Neuro-cardiac Interaction in Malignant Ventricular Arrhythmia and

\title{
Sudden Cardiac Death
}

G. André $\mathrm{Ng}^{1,2,3}$

1. Department of Cardiovascular Sciences, University of Leicester, Leicester, UK

2. National Institute for Health Research Leicester Cardiovascular Biomedical Research Unit

3. University Hospitals of Leicester NHS Trust, Glenfield Hospital, Leicester, UK

Professor G. André Ng

Department of Cardiovascular Sciences

University of Leicester, Glenfield Hospital,

Leicester, LE3 9QP, UK

Telephone: $+44(0) 1162583297$

Fax: $+44(0) 1162875792$

E-mail: gan1@le.ac.uk 


\begin{abstract}
Sudden cardiac death as a result of lethal ventricular arrhythmias is a major cause of death in cardiac diseases such as heart failure and prior myocardial infarct. Activity of the autonomic nervous system is often abnormal where sympathetic activity is upregulated and vagal activity reduced in these conditions. The abnormal autonomic state has been shown to be a strong prognostic marker of increased mortality and propensity to lethal arrhythmias, for which there is no effective prevention. Research effort over the years has established good evidence for a causal link between autonomic disturbance and ventricular arrhythmias. However, the detailed electrophysiological mechanisms by which ventricular fibrillation occurs are still not clear and molecular processes which are modulated by autonomic nerve influences that either predispose the heart to or protect it from these arrhythmias are not fully understood. This review presents data from studies investigating the link between activity of the autonomic nervous system and ventricular arrhythmias, from seminal findings in classical studies to ongoing investigations, in the quest for a better understanding of the arrhythmogenic mechanisms underlying neurocardiac interactions with a view to the development of effective preventative and therapeutic strategies which are very much needed.
\end{abstract}

Keywords: Sympathetic; vagus nerve; sudden cardiac death; ventricular arrhythmia; neurocardiac interactions; ventricular fibrillation; autonomic nervous system 


\section{Introduction}

Sudden cardiac death (SCD) claims around 90,000 lives each year in the UK and remains a major unsolved clinical problem. The majority of these deaths are due to malignant ventricular arrhythmias [ventricular tachycardia (VT) and fibrillation (VF)]. The current therapy with proven benefit is the implantable cardioverter defibrillator, which detects the high rates of VT/VF, and delivers either an electric shock or overdrive pacing to revert the arrhythmias back to normal rhythm to avert death. Implantable cardioverter defibrillators have been shown in well-conducted randomised clinical trials (Lee et al. 2003) to reduce arrhythmic and total mortality compared with antiarrhythmic drugs for both secondary prevention (i.e. in patients who have survived a sudden death event) and primary prevention (i.e. in patients who are deemed at high risk of sudden death). However, the treatment is crude and palliative and there is no preventative treatment strategy that is effective either in the form of device intervention or drugs. This is due to the fact that the mechanisms underlying these lethal arrhythmias are still poorly understood despite decades of research.

Multiple lines of evidence point to a pivotal role of the autonomic nervous system in the development and maintenance of arrhythmias (Shen \& Zipes 2014). ß-blockers have been shown to be of prognostic benefit in certain heart conditions, with data supporting some reduction in SCD mortality (Al-Gobari et al. 2013) highlighting an important association between sympathetic tone and ventricular arrhythmias. Left cardiac sympathetic denervation has been shown to be effective in treating and reducing arrhythmia burden in patients with refractory ventricular arrhythmias (Schwartz 2014). Epidemiological studies and studies in patients with implantable cardioverter defibrillators have shown that a high 


\section{Autonomic nervous system and cardiac arrhythmic death}

\section{Cardiac innervation}

Sympathetic and parasympathetic fibres innervate all regions of the heart with sympathetic fibres arising primarily from the thoracic vertebrae of the spinal cord with pre-ganglionic nerves distributed to various bilateral paravertebral ganglia (Randall \& Rohse 1956). Postganglionic fibres converge at the cardiac plexus - a group of nerves and blood vessels at the aortic arch, before innervating the heart. The somata of the parasympathetic efferent preganglionic neurones arise chiefly from the ventral lateral region of the nucleus ambiguous, and a smaller proportion from the dorsal motor nucleus and intermediate zone between these sites (Armour 1996). Pre-ganglionic fibres are carried within the Xth cranial (vagus) 
nerves and converge at several atrial and ventricular ganglionated plexuses which regulate cardiac function through postganglionic projections postganglionic in the atrial and ventricular tissues (Coote 2013).

\section{Autonomic nervous system and sudden cardiac death}

There is prominent circadian variability in SCD occurrence with a peak incidence during the waking hours in the morning (Willich et al. 1987) which corresponds to the abrupt reduction in heart rate variability during the same time period, signifying the surge in sympathetic activity and withdrawal of vagal activity (Bonnemeier et al. 2003). Cardiac diseases such as chronic heart failure (Nolan et al. 1998) and myocardial infarction (La Rovere et al. 1998) are associated with abnormal cardiac autonomic control including high levels of sympathetic activity, impaired parasympathetic control and subnormal arterial baroreflex sensitivity. Clinical measurements of autonomic tone, such as heart rate variability and baroreflex sensitivity, have been shown to be powerful and independent prognostic markers in these disease states and in survivors of cardiac arrest (Kleiger et al. 1987; Casolo et al. 1989; Dougherty \& Burr 1992). This relationship has led to the suggestion that impaired cardiac autonomic regulation does not merely reflect cardiac dysfunction but actively and adversely contributes to disease progression and mortality.

There is strong evidence that much of the relationship between impaired cardiac autonomic control and mortality is a result of an increased susceptibility to lethal ventricular arrhythmias (Schwartz 1998). Sudden arrhythmic death is a common mode of death in survivors of myocardial infarction and in chronic heart failure. Animal studies have shown that sympathetic stimulation via the stellate ganglia increases the vulnerability to ventricular fibrillation (VF) of the normal heart (Verrier et al. 1974). Acute ischaemia with 
coronary artery occlusion is associated with a surge in sympathetic discharge and has been shown to reduce the threshold for VF (i.e. increased susceptibility to VF), an effect which is prevented by pre-treatment with $\beta$-adrenergic blockers (Parker et al. 1990). In contrast, it has been known for over 100 years since the work of Einbrodt and his 'inductorium' that vagal stimulation increases the threshold for VF, i.e. reduced susceptibility to VF (Einbrodt 1859). Work in anaesthetised animals has confirmed this effect (Kent et al. 1973; Zuanetti et al. 1987). The seminal work by Schwartz and his group provided insight in the association between autonomic tone and arrhythmic death in a conscious canine model of sudden ischaemic cardiac death. It was found that concomitant vagus nerve stimulation significantly prevented ischaemia-induced VF (Vanoli et al. 1991). Of equal significance, it was found that baseline baroreflex sensitivity was high in animals resistant to VF and low in susceptible animals (Schwartz et al. 1988). These data suggest that high levels of vagal activity can exert powerful anti-arrhythmic effects, which can counter the effects of acute ischaemia and sympathetic activation. Kolman (Kolman et al. 1976), however, showed that vagal stimulation alone did not affect the VF threshold but prevented the decrease in VF threshold induced by simultaneous sympathetic stimulation. Others have noted that the anti-arrhythmic effects of vagal stimulation are most pronounced during sympathetic activation (Vanoli et al. 1991). The effects of sympathetic and vagal stimulation have a different time course of action and the interactions between the two are complicated and unpredictable (Levy 1997). Part of the reason for some of these conflicting data may be due to the fact that the experiments were carried out in vivo and interference from circulating catecholamines and other neurotransmitters cannot be excluded.

\section{Autonomic nervous system and Heart Failure}


The link between the autonomic nervous system and SCD is particularly evident in heart failure where it is acknowledged that the increased likelihood of SCD is associated with mechanical dysfunction as assessed by a low left ventricular ejection fraction. A classical hallmark of heart failure, and also in patients following an $\mathrm{MI}$, is abnormal autonomic control (or 'autonomic imbalance'), namely high sympathetic activity and low vagal tone which occurs in all aspects of cardiovascular regulation e.g. alterations of afferent signalling, central processing, ganglionic and efferent innervation in addition to alterations to the heart itself. Changes in autonomic activity appear early in heart failure and often precede other changes typical of the disease. Vagal tone significantly decreased 3 days after cardiac dysfunction and preceded sympathetic over-activity in a canine heart failure model (Ishise et al. 1998). In patients with cardiac dysfunction, sympathetic activation occurs before symptoms and reduced parasympathetic drive is present early in patients with symptomatic heart failure from even mild LV impairment (Grassi et al. 2001). Irrespective of the relative timing that autonomic dysfunction ensues in heart failure, there is strong evidence that impaired neurocardiac control is intimately linked with increased mortality as a result of lethal ventricular arrhythmias (Schwartz 1998). The clinical and prognostic significance of sympathetic over-activation forms the rationale for $\beta$-blockers as standard heart failure therapy, whilst parasympathetic regulation has received much less attention.

\section{The sympathetic nervous system and ventricular arrhythmias}

A direct link between sympathetic activity and ventricular arrhythmia was demonstrated in

a canine model of SCD where ventricular arrhythmia was preceded by instantaneous sympathetic activity from ambulatory left stellate ganglion recording (Zhou et al. 2008). 
Whilst it has been acknowledged that sympathetic activation is associated with increased ventricular arrhythmia initiation, the underlying mechanisms are still poorly understood.

\section{Ventricular arrhythmia mechanisms and sympathetic activation}

The electrophysiological mechanisms underlying ventricular arrhythmia can include the classical mechanisms of abnormal automaticity, triggered activity and re-entry, all of which can be enhanced with sympathetic activation. In patients with prior myocardial infarct, the "scar" area contains dead and surviving myocardium with cardiac magnetic resonance studies with late gadolinium enhancement suggesting that myocardial scar quantification can predict arrhythmic events (Gao et al. 2012). It is not difficult to comprehend that the ideal environment of dead tissue in the vicinity of surviving myocardium would create an excellent milieu for re-entrant arrhythmia, i.e. ventricular tachycardia (VT), given the right trigger or stimulus, such as critically timed ectopic beat(s) which can be enhanced with sympathetic activation. However, it should be noted that stable VT is distinct from VF. In the presence of relatively preserved ventricular function or when VT rate is not too fast, VT can remain haemodynamically stable, unless it degenerates into VF which when sustained is invariably unstable and causes cardiac arrest. The mechanisms underlying VF initiation are still poorly understood and how stable VT degenerates into VF is still not clear (Weiss et al. 2000). Clinical trials have provided important information that tests used to induce VT in patients with prior $\mathrm{MI}$ and low left ventricular ejection fraction would predict future VT events but not necessary VF in patients with implantable cardioverter defibrillators (Daubert et al. 2006). VF can arise without going through a period of stable VT and hence its initiation requires better understanding.

\section{Dispersion of refractoriness}


A major direct electrophysiological effect of the autonomic nervous system on the ventricular myocardium is that on the recovery of excitability. Sympathetic stimulation has been shown to shorten whilst vagal stimulation lengthens the ventricular refractory period (Martins \& Zipes 1980). Substantial sympathetic-parasympathetic interaction also occurs in the effect on ventricular refractoriness (Nattel et al. 1981; Morady et al. 1988) but the mechanism behind the increase or decrease in arrhythmogenic tendency is not well understood.

The pioneering work of Han and Moe (Han \& Moe 1964) demonstrated that dispersion of recovery was increased by conditions that predispose to VF, e.g. sympathetic stimulation and cardiac glycoside toxicity; and that the increased dispersion was associated with increased VF inducibility. Elegant experiments by Kuo et al. (Kuo et al. 1983) further detailed the initiation of re-entrant ventricular arrhythmias with a critical level of dispersion of monophasic action potential duration. It is logical to extend the proposal to the importance of pre-existing heterogeneity in refractoriness to sustain VF in diseased hearts, which can be exaggerated with sympathetic stimulation to increase the propensity to ventricular arrhythmias. Further work on the dynamics of fibrillation in mathematical modelling investigations using more realistic ionic models of cardiac tissue demonstrated that fibrillation can occur in the absence of pre-existing heterogeneity in refractoriness (Panfilov \& Holden 1990). In a homogeneous sheet of cardiac tissue, it was shown that a stable reentrant or 'spiral' wave can, after a few rotations, "break" around the core and produce a daughter wave which can again break to give more wavelets. This provides a different view to the original multiple wavelet hypothesis proposed by Moe where the re-entry waves arise from heterogeneities in refractoriness. Here, spiral breakup of the rotating waves 
occurs without pre-existing non-uniformity of refractoriness. An alternative explanation is required for the conceptualisation of wavebreak and does sympathetic activation exploit this mechanism to promote arrhythmogenesis?

\section{Action Potential Duration Restitution and wavebreaks}

It is a normal intrinsic property of the action potential duration (APD) of myocardium to vary according to the activation rate. At slow heart rates, APD is long and with faster heart rates, APD shortens (Figure 1A \& B). This property is termed APD restitution. This is important mechanically where the cardiac cycle is shortened at faster heart rates to allow brisker contraction and relaxation for the efficient function of the cardiac pump. APD restitution is also not a novel concept but is embedded in the need for rate correction of the QT interval (surrogate of ventricular APD) on ECG with the recognition of the relationship between QT and heart rate (Ahnve 1985). The APD is dependent on the preceding diastolic interval (DI) and APD restitution describes the situation whereby a shortening of the DI leads to a shortening of the following APD. The relationship can be described by plotting APD against DI which usually assumes an exponential curve (Figure 1C). At slow heart rates, i.e. long DI, changes in DI lead to relative little change in APD with a flat APD-DI relationship. During restitution, APD shortens as DI decreases. At the steep part of the APD restitution curve, small changes in DI would lead to large changes in APD. The importance of the dynamics of the relationship between APD and DI was appreciated as early as 1968 when Nolasco and Dahlen (Nolasco \& Dahlen 1968) demonstrated in frog ventricular muscle strips that APD restitution slope increased when stimulus frequency increased with transient alternation in APD lasting longer. At a driving rate where APD restitution slope was +1 , persistent alternans occurred. This was interpreted graphically in the light of a feedback mechanism, 
which is illustrated in Figure 2. If the APD restitution relationship is shallow with a slope $<1$, a brief perturbation e.g. as a result of an ectopic beat will lead to a shorter DI, resulting in a shorter APD. Assuming that the heart rate is maintained constant i.e. cycle length $(\mathrm{CL}$, the sum of APD and DI) is constant and this relationship can be plotted as a straight line with the formula $A P D=-D I+C L$ and a slope of -1 . The following $D I$ will be longer, which makes the next APD less short as a result of the flat APD restitution curve and a stabilisation of the APD oscillations (Figure $2 A$ ). On the other hand, if the APD restitution slope is $>1$, a shortening in DI, will lead to more pronounced APD shortening, with amplification of effects on subsequent DI prolongation and setting up of APD alternans (Figure 2B). Thus the negative feedback if APD restitution slope is $<1$ and positive feedback if APD restitution slope is $>1$ serve to dampen or exaggerate oscillations and alternans respectively. It was shown that APD alternans can lead to breakup of spiral waves into a fibrillatory state in a two dimensional tissue simulation without spatial inhomogeneities (Karma 1993). The role that APD restitution slope plays in the generation of fibrillation through wave break was subsequently verified by biological data (Riccio et al. 1999; Garfinkel et al. 2000). It was first shown in arterially perfused canine ventricular endocardial preparations that drugs that reduce the APD restitution slope (diacetyl monoxime (DAM) and verapamil) prevented the induction of VF whilst the drug procainamide which does not reduce APD restitution slope did not prevent VF induction (Riccio et al. 1999). More spectacularly, DAM and verapamil converted VF into stable VT whereas procainamide did not lead to spatiotemporal organisation of VF. Similar findings were shown with bretylium in perfused swine right ventricular preparations, in which there was flattening of APD restitution curve, prevention of wavebreak and thus fibrillation induction and also converted VF into a periodic rhythm (Garfinkel et al. 2000). These studies provide strong experimental support for the 
"restitution hypothesis" and opened up lines of investigations both to explore the importance of APD restitution in mechanisms underlying arrhythmogenic conditions and also the possibility of manipulating APD restitution to reduce wavebreak and prevent or treat fibrillation.

\section{Sympathetic nerve stimulation, APD restitution and VF}

In order to investigate the mechanisms underlying the autonomic modulation of VF, our group developed an in vitro Langendorff perfused innervated rabbit heart preparation ( $\mathrm{Ng}$ et al. 2001b) which allows controlled stimulation of autonomic nerves without the confounding influence of underlying tonic autonomic activity, haemodynamic reflexes, circulating hormones or anaesthetics. Stimulating sympathetic inputs from within the spinal cord, reduced the stimulus current required to induce VF i.e. the VF threshold (VFT), indicating an increased susceptibility to VF. The slope of the electrical restitution curve was increased (Ng et al. 2007) suggesting a causal link between APD restitution and VFT (Figure 3). The increase in APD restitution slope has also been shown in human patients using adrenergic agonists isoprenaline or adrenaline (Taggart et al. 2003).

\section{Electrophysiological effects of sympathetic activation}

One strategy to address the pro-arrhythmic effect of sympathetic activation would be to target the specific electrophysiological effects, which requires an understanding of the underlying mechanisms. Sympathetic activation generally shortens ventricular refractoriness and APD (Martins \& Zipes 1980). This is believed to be mainly mediated via outward (in particular $\mathrm{K}+$ ) currents which are sensitive to adrenergic stimulation, e.g. slow delayed rectifier K+ current, IKs (Volders et al. 2003). This mechanism may also be a 
contributory factor to the steepening of the APD restitution curve with sympathetic activation, which implies an increase in outward current or reduction in inward current. However, other important ion channels are also affected by sympathetic activation, especially Ca2+ channels, which can significantly alter [Ca2+] levels and engage other arrhythmogenic mechanisms such as triggered activity with early / delayed afterdepolarisations (Pogwizd \& Bers 2004). The precise contributions of these various mechanisms are not well understood.

\section{Laterality and heterogeneous innervation}

Whilst the heart requires many processes to be co-ordinated to work efficiently, there is a degree of natural heterogeneity within the organ and its associated systems which when altered could have implications in arrhythmogenesis. There is consensus that unilateral stimulation of sympathetic input to the heart can result in differential effects. Pioneering studies by Walter Randall's group (Ardell et al. 1988) demonstrate that right sided sympathetic stimulation increases heart rate to a larger extent that the left, whilst the left sided input preferentially affects the atrioventricular node and increases left ventricular force to a greater extent than the right. These 'laterality' differential effects are extended to ventricular electrophysiological effects where stimulation / ablation of either stellate ganglia has differing effects on refractoriness, ECG and VF induction (Haws \& Burgess 1978; Yanowitz et al. 1966; Schwartz et al. 1976). Removing the right stellate ganglia or left sided sympathetic nerve stimulation (SNS) is associated with an increased susceptibility to VT with a greater occurrence of early and delayed afterdepolarisations, whilst right SNS has less of an effect (Priori et al. 1988). The detailed innervation pathway from different sides of the sympathetic chain and ganglia to the heart and the resultant cardiac electrophysiological 
effects from their activation have not been fully described. Differential intra-ventricular effects also exist, with left SNS resulting in a greater reduction of APD at the base of the left ventricle (LV) (Winter et al. 2012). This has important clinical implications as left thoracic sympathetic denervation may serve as effective anti-arrhythmic therapy in post-infarct patients (Schwartz et al. 1992), long QT Syndrome (Schwartz et al. 2004), catecholaminergic polymorphic VT (Wilde et al. 2008) and VT storm (Bourke et al. 2010).

The differences in the functional response to SNS can be due to heterogeneous sympathetic innervation or ion channel distribution, with the resultant ventricular electrophysiological effects. It is known that catecholamine levels are higher at base of the LV (Dahlström et al. 1965) whilst there is a significant base $\rightarrow$ apex distribution of sympathetic nerves in animal and human hearts (Kawano et al. 2003; Ng et al. 2009). The greater basal APD shortening during SNS may significantly alter the spatial properties of ventricular restitution and increase heterogeneity of repolarisation, which is a well-recognised substrate for arrhythmia initiation (as described above). During sinus rhythm it is known that SNS reverses the direction of repolarisation (Mantravadi et al. 2007), from an apex $\rightarrow$ base direction to a base $\rightarrow$ apex, owing to the differential response at the LV base. Figure 4 illustrates APD plotted against DI during SNS and compared to the same changes in heart rate produced with pacing but without underlying nerve stimulation. There was greater APD shortening seen with sympathetic nerve stimulation, being more prominent at the left ventricular base where there was an increased distribution of sympathetic nerves and of the slow delayed rectifier K+ channels (IKs). Transmurally, SNS can also promote triggered activity (delayed afterdepolarisations) within the LV mid-wall (Zhang et al. 2004). 
The issue with heterogeneity is made more complex in pathological states whereby following myocardial infarct, there is both denervation (Kammerling et al. 1987) and regional hyperinnervation (Cao et al. 2000) seen, with sympathetic nerve sprouting (Zhou et al. 2004) and increased variability in regional responses which has been linked with increased arrhythmogenicity. In addition to myocardial remodelling that occurs in pathological states following myocardial infarct or in heart failure, there is also evidence of remodelling in the stellate ganglion neurons (Han et al. 2012; Ajijola et al. 2015) and intrinsic cardiac neurons (Rajendran et al. 2016) which adds to the complexity encouraging instability in the already arrhythmogenic environment.

\section{The parasympathetic nervous system and ventricular arrhythmias}

Vagal post-ganglionic nerves primarily modulate cardiac activity through the release of acetylcholine acting on post-synaptic muscarinic receptors (mAChRs) to slow heart rate (bradycardia), delay atrioventricular conduction (dromotropy) and reduce atrial force (inotropy) with little effect on ventricular performance. The lack of evidence to support a direct negative ventricular inotropic effect reflects historical data which suggested against a significant cholinergic innervation in the ventricle. This view is however outdated (Coote 2013) as modern techniques reveal a dense and intricate network of acetylcholinecontaining nerves running over the epi- and endo-cardial surfaces of left and right ventricles and a widespread distribution of mAChRs throughout the ventricle (Brodde et al. 2001). More importantly, it is worth noting that the vagal-cholinergic pathway is capable of inhibiting the strong inotropic response to adrenergic activation (Brack et al. 2010) and as such has a clear functional role. Dense parasympathetic innervation is demonstrated in all species studied including the mouse (Rysevaite et al. 2011), guinea pig (Batulevicius et al. 
2005), rabbit (Pauziene et al. 2016), cat (Johnson et al. 2004), dog (Pauza et al. 2002), pig (Ulphani et al. 2010), sheep (Saburkina et al. 2010) and human ventricle (Pauza et al. 2000; Kawano et al. 2003).

\section{Vagal modulation of ventricular arrhythmia susceptibility}

Classical and more recent studies have consistently shown that stimulation of the vagus nerve protects the heart against VT / VF or reduces their incidence. These studies have been summarised in a previous review (Brack et al. 2013). The first report that vagus nerve stimulation (VNS) reduced the susceptibility of the ventricle to experimental arrhythmia was presented by Einbrodt in 1859 (Einbrodt 1859), who demonstrated that VF was harder to induce in the dog during stimulation of the vagus. The anti-arrhythmic action of VNS was later reproduced by Scherlag's group (Scherlag et al. 1970) who showed that VNS interrupted the spontaneous occurrence of VT during coronary artery occlusion . Other studies in the 1970's (Goldstein et al. 1973; Myers et al. 1974; Kent et al. 1973) confirmed the anti-arrhythmic action of VNS in the ventricle during ischaemia. Several studies provided evidence that VNS is also protective against experimental induced VF - with the demonstration of a VNS dependent increase in the current required to induce VF, i.e. the ventricular fibrillation threshold (VFT), in the anaesthetised dog (Kolman et al. 1975; Yoon et al. 1977). However, it was shown that the VNS-induced increase in VFT was not maintained in the presence of the non-selective $\beta$-adrenergic antagonist propranolol (Yoon et al. 1977). Moreover, the anti-arrhythmic effect with VNS was only seen with concomitant sympathetic nerve stimulation and was lost when the left stellate ganglion was crushed (Kolman et al. 1975), supporting significant sympathetic-parasympathetic interactions in the antiarrhythmic action of VNS, which are discussed in later. 


\section{VNS, ventricular arrhythmias and myocardial ischaemia}

Coronary artery occlusion with concurrent myocardial ischaemia is well-recognised as a potent trigger of arrhythmia and major cause of fatality in myocardial infarction. There is evidence from in vivo studies under anaesthesia in experimentally induced VF, that VNS is protective under these conditions (Scherlag et al. 1970; Goldstein et al. 1973). Conversely, bilateral vagotomy or atropine perfusion increases arrhythmic mortality in anaesthetised cats during (Corr \& Gillis 1974) and following coronary artery occlusion (Zuanetti et al. 1987) suggesting that intrinsic vagal activity was protective. In contrast, there was some conflicting data reporting no effect of vagal stimulation on VFT in the ischemic dog heart despite a pronounced response in non-ischaemic controls (Yoon et al. 1977).

To overcome the confounding influence of anaesthetics in experimental animal studies, Peter Schwartz and his group developed an elegant model of SCD in conscious dogs which had been used extensively to study the protective effect of the vagus in conscious animals (Schwartz et al. 1984). In this model, a myocardial infarct is produced via a left anterior descending coronary artery ligation and animals are instrumented with a balloon (to occlude the left circumflex artery at a later date). Animals are allowed to recover for 1 month and surviving animals then have a treadmill exercise test with increasing workload. During the last stage of exercise, the left circumflex coronary artery is occluded for a period of 2 minutes. This combination of adrenergic activation and myocardial ischaemia results in a reproducible and quantifiable measure of SCD, with VF occurring in $50-60 \%$ of all post-MI dogs (Schwartz et al. 1988). In the majority of dogs that survived, there was a reduction in heart rate, suggesting that active vagal reflexes are protective and was later confirmed when the group (De Ferrari et al. 1991) directly measured baroreflex sensitivity and 
demonstrated that protection against ischaemia-induced VF was significantly associated with indirect measures of preserved vagal reflexes. The authors also found that muscarinic blockade with atropine promoted premature ventricular contractions, VT and VF in animals with no arrhythmia during control. The group also studied the effects of direct VNS on the occurrence of SCD in dogs that survived MI (Vanoli et al. 1991). Right VNS, applied before and throughout coronary artery occlusion, reduced the occurrence of VF from $92 \%$ in control to $10 \%$ during VNS. These data provide strong supportive evidence of the important anti-arrhythmic protection of the vagus nerve on the ventricle.

\section{Direct anti-VF protection - studies in the isolated innervated rabbit heart}

Whilst VNS has been shown to protect the heart against ventricular arrhythmias, the in vivo studies and the focus on ischaemia induced arrhythmia made it difficult to elucidate if these were due to direct effects of VNS as it is unclear if there was adequate $\beta$-blockade and as such these data may reflect indirect effects. Using the innervated heart preparation $(\mathrm{Ng}$ et al. 2001b), our group studied the effects of VNS on electrical restitution and VFT (Ng 2014). VNS caused a flattening of the electrical restitution slope whilst simultaneously increasing VFT (Ng et al. 2007) with prolongation of monophasic APD and effective refractory period (Figure 3). VFT was inversely correlated with the slope of the APD restitution curve, in keeping with the restitution hypothesis and the effects occurred in the absence of background sympathetic tone strongly supporting a direct anti-arrhythmic action.

\section{Mechanisms underlying the anti-arrhythmic effect of vagus nerve stimulation}

Over the years, the vagus nerve has been found to do a lot more than just slowing down the sinus rate and its stimulation has far-reaching effects in many processes related to cardiac 
function (Olshansky et al. 2008) some of which may play an important role in protecting the heart against ventricular arrhythmias (Brack et al. 2013) (Figure 5).

\section{Accentuated antagonism}

In the intact organism, both divisions of the autonomic system are tonically active. At rest, vagal tone predominates, whilst sympathetic tone predominates during the day. In disease situations such as myocardial ischaemia and heart failure, activity of both systems is disturbed (see earlier). This is important because sympathetic and parasympathetic nerves interact with one another. It was recognised in the 1930's that vagus nerve stimulation (VNS) attenuated the chronotropic enhancement associated with sympathetic stimulation (SNS), with the actual change in heart rate with concomitant VNS and SNS being different from the algebraic sum of the heart rate changes during individual stimulation of each branch (Samaan 1935). This interaction was later coined 'accentuated antagonism' by Matthew Levy (Levy \& Zieske 1969) who showed that the vagus exerts a powerful antagonistic effect on the chronotropic actions of SNS, data which were confirmed by others, including our group on the innervated rabbit heart preparation (Brack et al. 2004). A similar antagonistic action has been reported for the control of ventricular performance and intracellular calcium handling (Levy et al. 1966; Brack et al. 2010). With regard to arrhythmogenesis, it was found in anaesthetised dogs during ischaemia that VNS protection was only evident during simultaneous SNS (Kolman et al. 1975), as mentioned above. Similarly, the anti-arrhythmic effect of the cholinergic agonist methylcholine was augmented during norepinephrine perfusion (Rabinowitz et al. 1976). Interestingly, the protective effect of VNS was reported to be abolished during coronary artery occlusion and propranolol administration (Yoon et al. 1977), although this is at odds with other studies. The abolition of VNS mediated protection during ischaemia may however imply that 
sympathetic activity could reduce the effect of the vagus because reflex sympathetic activity is increased with ischaemia. At the cellular level, interaction between sympathetic and vagal nerves can occur via pre- and / or post-junctional mechanisms. As an example, for effects of vagal activity on sympathetic stimulation, acetylcholine binds to muscarinic acetylcholine receptors (mAChRs), primarily the $\mathrm{m} 3$ subtype present on the pre-synpatic sympathetic nerve terminals to inhibit noradrenaline release and principally the $\mathrm{m} 2$ subtype on cardiac myocytes to modulate intracellular cAMP at a number of stages (Muscholl 1980). Conversely, the downstream effects of vagal stimulation via acetylcholine release can be modulated post-synaptically by sympathetic activity through noradrenaline and co-released neuropeptide Y (Potter \& Ulman 1994; Herring et al. 2008).

\section{Heart rate reduction and ventricular refractoriness and APD dispersion}

An obvious aspect to consider regarding the antiarrhythmic protection during VNS is the resultant bradycardia. Reducing heart rate using the $I_{f}$ blocker ivabradine increases VFT (Vaillant et al. 2008) suggesting that the effect of VNS may be due to heart rate. This should however be regarded with caution as there is recent evidence that ivabradine can have effects on ventricular repolarisation (Melgari et al. 2015) and may have other 'antiarrhythmic' effects (Koncz et al. 2011) as opposed to a pure effect on heart rate alone. Nonetheless, the protective effect of VNS during coronary artery occlusion in the postinfarct canine exercise experiments was either reduced (Goldstein et al. 1973) or abolished (Schwartz et al. 1984) when heart rate was controlled. VNS prevented VF in $55 \%$ of animals that were rate controlled vs. $92 \%$ of control dogs in sinus rhythm, suggesting that bradycardia 'is important but not essential'. We have shown that the anti-VF effect of VNS is preserved during constant pacing in the isolated innervated rabbit heart (Ng et al. 2007). In addition, we have shown that despite differential heart rate effects of left and right VNS, the 
anti-fibrillatory effect is equipotent (Ng et al. 2001a). The mechanisms by which a reduction in heart rate could protect from ventricular arrhythmias is not understood, but may include rate-dependent alterations in ventricular APD, refractoriness and dispersion of both factors. It is recognised that VNS prolongs ventricular APD and effective refractory period (Martins \& Zipes 1980; Ng et al. 2007; Mantravadi et al. 2007) which are inversely correlated to heart rate, and that slower heart rates decreases the dispersion during ischaemia (Kent et al. 1973). Spatial repolarisation heterogeneity provides a substrate for the generation of ventricular arrhythmias and dispersion of APD is a known mechanism underlying circus movement re-entry around refractory regions (Kuo et al. 1985). Although previous investigators have noted that the effects of VNS on electrophysiology are uniformly distributed (Martins \& Zipes 1980), with no effects of APD dispersion, it is known that the SNS promotes a greater degree of APD shortening in basal regions of the ventricle (Ng et al. 2009), data which is supported by histological studies of sympathetic nerve distribution (Kawano et al. 2003). When there is background sympathetic tone, VNS may prevent arrhythmias by antagonising sympathetic mediated changes in spatial repolarisation (see accentuated antagonism). This idea may be particularly relevant in disease states such as heart failure where there is an increased activation and influence of the sympathetic nervous system.

\section{Nitric Oxide (NO) and APD restitution}

Muscarinic acetylcholine receptors (mAChRs) are activated by acetylcholine and there are five subtypes (m1-5) with $\mathrm{m} 2$ being the dominant subtype in the heart. A reduction of arrhythmia protection using the $\mathrm{mAChR}$ antagonist atropine in earlier studies suggests that mAChR activation is important in the modulation of antiarrhythmic property of vagus nerve stimulation. In autonomically-intact dogs, spontaneous ischaemia-induced VF increased in 
vivo following i.v. atropine (Goldstein et al. 1973), with similar conclusions drawn from control experiments in the dog model of SCD (Schwartz et al. 1984; Vanoli et al. 1991). This is supported by data during direct VNS where atropine abolished protection (Yoon et al. 1977). The mAChR agonist choline is cardioprotective against ischaemia induced arrhythmias, an effect that is blocked by the m3-AChR antagonist 4-DAMP (Wang et al. 2012) supporting the importance of muscarinic activation in the tentative protective effect of parasympathetic activation against ventricular arrhythmias. However, this is at odds with our findings in the innervated heart preparation, where the protection of VNS against VF was preserved in the presence of atropine despite the effects of bradycardia and prolongation of effective refractory period being blocked (Brack et al. 2011).

NO is simple free radical gas generated by a family of enzymes known as NO synthases (NOSs) of which there are 3 isoforms - the constitutive calcium-dependent nNOS (NOS1) and eNOS (NOS3) and the calcium-independent, cytokine-inducible iNOS (NOS2). nNOS is mainly found in neuronal tissues while eNOS is present in vascular endothelium, platelets, myocardium and endocardium. iNOS is expressed in macrophages and other tissues with immune stimulation. The important role of NO in the cardiovascular system was first demonstrated in the mediation of acetylcholine-induced vasodilatation (Furchgott \& Zawadzki 1980; Ignarro et al. 1987; Palmer et al. 1987) but it is now known that NO mediates many aspects of cardiac function at the organ level (Kelly et al. 1996). NO production is also intimately involved in the neural control of the cardiovascular system (Zanzinger 1999) in both central and peripheral aspects of vagal control. There are some parallels to the control in other bodily systems such as the gastrointestinal tract (Grundy \& Schemann 2007). In the heart NO has been shown to modulate the actions of the 
parasympathetic nervous system, regulating the change in heart rate in response to parasympathetic stimulation (Conlon \& Kidd 1999) with data supporting a pre-synaptic action to reduce the bradycardic response to VNS (Herring et al. 2000). It was also noted that NO modulates changes in A-V conduction in response to VNS in a similar manner (Conlon \& Kidd 1999) proposing that NO may have a role in the ventricular effects of VNS. We directly studied this using the innervated rabbit heart preparation .

Our group has been investigating the role of $\mathrm{NO}$ in mediating the vagal protection against VF initiation in the isolated innervated heart preparation especially in relation to the effects on APD restitution as electrophysiological mechanism ( $\mathrm{Ng}$ 2014). We first established in the preparation that VNS caused a flattening of the APD restitution slope, increased effective refractory period and increased VFT (Ng et al. 2007) (Figure 3). In the presence of the nonspecific nitric oxide synthase (NOS) inhibitor NG-nitro-L-arginine (L-NA), the increase in VFT and flattening of the APD restitution slope with VNS were abolished, whilst the increase in effective refractory period was preserved (Brack et al. 2007) (Figure 6). The response to VNS was restored by supplementation with L-arginine, the NO substrate which competes with LNA for binding to NOS. These data tentatively show that APD restitution is important as a mechanism underlying the protection from VF provided by VNS and that NO plays a key role in this protective effect. The protective effect of vagally-released NO is supported by data using NO donors in other studies. Nitro-glycerine epicardially applied during coronary artery occlusion (Kumar et al. 2000) or sodium nitroprusside perfusion (Kalla et al. 2016) during experimentally induced VF in nonischaemic hearts mimics the effect of VNS. However, the experiments provided only indirect evidence. Through use of the NO-sensitive dye DAF-2 DA, our group developed a novel fluorescence technique (Patel et al. 2008) for measuring 
NO in the whole heart and were able to provide, for the first time, direct evidence of NO release in the ventricle resulting from VNS (Brack et al. 2009) (Figure 7). NO-dependent fluorescence increased during both left and right cervical VNS with the intensity of stimulation. Perfusion of L-NA significantly decreased background fluorescence, indicative of a reduction in basal NO production (presumably from the endothelium), and abolished the increase in fluorescence seen with VNS. TRIM, a specific antagonist of the neuronal form of NOS (nNOS or NOS I), had no effect on background fluorescence but abolished the increase in fluorescence with VNS, supporting the notion that NO production during VNS occurs through nNOS activation (Brack et al. 2009). Following this, we have extended these findings to show that these effects of the vagus do not occur through muscarinic activation, because perfusion with atropine at a concentration sufficient to block changes in heart rate did not influence the vagal effect on DAF-2 fluorescence, the reduction of the slope of the electrical restitution curve or the increase in VFT (Brack et al. 2011). In addition, this study indicates that the protective effects do not involve the endothelium (a substantial source of NO in the heart), because effects are preserved after functional endothelial denudation or during inhibition of vasoactive intestinal peptide, which is a neuroactive peptide released together with acetylcholine (Henning et al. 2001). It has previously been suggested that vagally released acetylcholine and NO can act through parallel independent signalling pathways (Jumrussirikul et al. 1998). Given our evidence that NO production during VNS occurs through nNOS independent of the endothelium, vasoactive intestinal peptide or acetylcholine, it would support the existence of a separate network of parasympathetic nitrergic antifibrillatory neurons within the ventricle (Jones 2009). It is known that nNOS is present in many parasympathetic neurons innervating the heart, and there is also evidence 
of a subpopulation of intracardiac nerve fibres that solely contain NO, coursing towards the ventricle in humans (Hoover et al. 2008), providing supportive evidence for our hypothesis.

\section{Anti-inflammatory action}

Over the past 15 years, it has become increasingly evident that the inflammatory response is under control by the autonomic nervous system in a process termed 'the inflammatory reflex' (Tracey 2002). Upon activation, locally released inflammatory cytokines such as tumor necrosis factor (TNF) activate sensory nerves relaying information to central processes. After processing, efferent activity is altered to regulate the injury response. Vagotomy, not only augments the cytokine response to injury but heart rate variability is also inversely correlated to cytokine levels (Sloan et al. 2007). Lower levels of cytokines are generally thought to be beneficial to restrict the inflammatory response following myocardial ischaemia, heart failure and myocarditis - where arrhythmias contribute significantly to mortality. How the inflammatory reflex regulates arrhythmia is not understood but may involve both direct anti-arrhythmic actions and indirect effects which ameliorate disease related structural and biochemical processes. VNS improves survival following coronary artery occlusion by reducing infarct size (Katare et al. 2009; Calvillo et al. 2011) with beneficial increases in myocardial ATP, reduced levels of apoptosis and cell swelling via inhibition of the mitochondrial permeability transition pore (Katare et al. 2010). This is blocked by atropine with subsequent up-regulation of TNF- $\alpha$ and activation of the cell survival TNF receptor 2 , nuclear factor kappa-B and pAkt pathways. In macrophages, TNF- $\alpha$ can promote connexin-43 expression and gap junction formation (Eugenin et al. 2003). This could correct ischaemia induced conduction abnormalities and is increased during VNS which was more importantly linked to the protection of ischaemia induced VT (Ando et al. 2005). The significance of inflammation in chronic heart failure is somewhat unclear, 
although serum and cardiac levels of inflammatory cytokines are increased. Their levels are significantly reduced during VNS (reviewed in (Li \& Olshansky 2011)). The reduction of cytokines is suggested to occur though acetylcholine acting on nicotinic acetylcholine receptors (nAChRs), primarily the $\alpha-7$ subtype, on macrophages (Wang et al. 2003). However, $\alpha-7$ nAChRs are also found on intracardiac nerves and cardiac myocytes (Dvorakova et al. 2005) raising the possibility that extra-macrophage $\alpha-7$ nAChRs may be involved in reducing inflammation in addition to mediating the functional effects of autonomic nerves (Deck et al. 2005). Genetic up-regulation of cardiac TNF- $\alpha$ is used in experimental heart failure models, highlighting its pathological significance. These animals show increased occurrence of arrhythmias (London et al. 2003). Hearts from these animals had prolonged APD and smaller potassium currents (Petkova-Kirova et al. 2006) and dysregulated intracellular calcium homeostasis leading to altered contractile function, mirroring those seen in clinical heart failure. In addition, cytokines indirectly increase arrhythmias by promoting the formation of non-conductive scar and fibrotic tissue (Klein et al. 2000).

\section{Indirect antiarrhythmic and non-arrhythmic beneficial effects of VNS}

There has been significant research effort spent in preclinical and clinical arenas exploring the utility of VNS in the chronic setting in heart failure to improve cardiac and haemodynamic function. Any potential changes in cardiac or overall function may also have important impact on ventricular arrhythmogenesis. The effects of chronic vagal stimulation on arrhythmia occurrence were investigated in conscious rats with $\mathrm{MI}$-induced heart failure (Zheng et al. 2005) where right cervical VNS conducted 3-5 months post-MI for a period of 7 days prevented the occurrence of premature ventricular and supraventricular contractions. The effects of chronic VNS were studied in dogs with microembolism induced heart failure 
(Sabbah et al. 2011) in which VNS was applied using a negative-feedback loop to maintain heart rate at a level $10 \%$ below baseline for a 3-month period. While the main focus of this study was on haemodynamics and ventricular remodelling, the effect of VNS on gap junction protein expression was documented. Heart failure causes translocation of the gap junction protein connexin away from the intercalated discs which regulate cell-to-cell excitability allowing the passage of small ions between adjacent cardiomyocytes. Disruptions to this cell-to-cell coupling play an important role in arrhythmogenesis and altered conduction through the myocardium. Reduced expression of connexin-43, the primary form expressed in the ventricular myocardium, slows ventricular conduction and increases the dispersion of transmural APD which is linked to the increased incidence of tachyarrhythmias and SCD (Ai \& Pogwizd 2004; Wang \& Gerdes 1999). VNS therapy reverses the reduction in connexin-43 mRNA and protein expression associated with heart failure (Sabbah et al. 2011) providing indirect evidence that VNS may have additional anti-arrhythmogenic properties in the failing heart via these mechanisms.

The effects of VNS can be mimicked by the use of pharmacological agents. Pyridostigmine, a cholinesterase inhibitor decreases the rate of acetylcholine degradation at the postganglionic synapse and prevents the occurrence of single ventricular ectopic beats in patients with New York Heart Association class I to III heart failure symptoms and left ventricular ejection fraction less than $45 \%$, although no effect was noted on paired ectopic beats or on the occurrence of periods of ventricular tachycardia (Behling et al. 2003). The suppression of cardiac arrhythmias in congestive heart failure by clonidine (Zhang et al. 1998), a centrally acting $\alpha 2$ adrenoceptor agonist which acts to suppress sympathetic 
nervous activity, demonstrates how VNS could be used to antagonise the pro-arrhythmic influence of the sympathetic nervous system.

Despite limited data on the effects of VNS on arrhythmia susceptibility there is good evidence that VNS improves autonomic balance in heart failure in animals studies (Li et al. 2003; Hamann et al. 2013; Zhang et al. 2009; Zhang et al. 1998) reducing sympathetic hyperactivity and increasing vagal tone, highlighting its potential as an anti-arrhythmic therapy. In addition to its anti-arrhythmic properties, chronic VNS has been shown to reverse the contractile dysfunction and adverse remodelling associated with experimental heart failure and appears to be beneficial even when applied at sub-threshold intensities insufficient to cause changes in HR (De Ferrari \& Schwartz 2011). These promising preclinical results have spurred on the enthusiastic development of clinical tools to apply VNS in patients with heart failure. This was facilitated by the implantable vagus nerve stimulator technology either already available for the treatment of intractable epilepsy (Binnie 2000) or the development of dedicated vagus nerve electrode and stimulator directed towards the heart (Anholt et al. 2011). A pilot study in 32 New York Heart Association Class II-IV patients showed promise with VNS via an implanted stimulator shown to be safe and feasible, reproducing much of what the animal studies showed i.e. significant improvements in New York Heart Association Class, quality of life, 6-minute walk test, left ventricular ejection fraction and LV systolic volumes which were maintained at 1 year (De Ferrari et al. 2011). This was followed by several larger clinical trials including NECTAR-HF (Zannad et al. 2015) and INOVATE-HF (Gold et al. 2016) which produced, unfortunately, disappointing results. The NECTAR-HF trial (with 96 patients implanted with VNS stimulators) failed to reproduce the functional improvement shown in the previous pilot study, albeit with different 
technology, whilst there was some symptom improvement. The INOVATE-HF trial (with 436 patients implanted with VNS stimulators) designed as an outcome trial failed to show benefit of VNS in the hard endpoints of death and heart failure events whilst there again appeared to be some subjective symptomatic improvement. A smaller ANTHEM-HF trial (Premchand et al. 2014) (with 60 patients implanted with VNS stimulators) showed some positive results similar to the initial pilot study. These clinical results have significantly reduced the momentum, which was built in the clinical translation of VNS in patients with heart failure and hence would not help in addressing the question whether VNS has a favourable effect on ventricular arrhythmia events. This would require an alternative clinical approach or different design of clinical studies.

\section{Conclusions}

Sudden cardiac death as a result of malignant ventricular arrhythmias remains a major unsolved clinical problem. Abnormal autonomic activity is the hallmark in cardiac diseases and there is strong evidence of an important association between the autonomic nervous system and the occurrence of ventricular arrhythmias although the precise mechanisms remain to be clarified. The significant role that autonomic modulation plays in the initiation and maintenance of ventricular arrhythmias and the possibility that nerve stimulation (as opposed to pharmacological analogue manipulation) could be exploited as a novel therapeutic modality has only received recent attention. Much more needs to be known in relation to the cellular mechanisms and downstream pathways. The autonomic nervous system may hold the key to the prevention and successful treatment of these important arrhythmias. 


\section{Acknowledgement}

Work described above has been supported by British Heart Foundation Project Grants (PG/99008, PG/02/088, PG/09/039), Garfield Weston Trust Project Grant and National Institutes of Health, USA, Grant (RO1 HL57929). The author wishes to acknowledge the administrative assistance from Miss Zhia N Lim. 


\section{References}

Ahnve, S., 1985. Correction of the QT interval for heart rate: Review of different formulas and the use of Bazett's formula in myocardial infarction. American Heart Journal, 109(3 PART 1), pp.568-574.

Ai, X. \& Pogwizd, S.M., 2004. Connexin 43 Downregulation and Dephosphorylation in Nonischemic Heart Failure Is Associated With Enhanced Colocalized Protein Phosphatase Type 2A. Circulation Research, 96(1), pp.54-63.

Ajijola, O.A. et al., 2015. Remodeling of stellate ganglion neurons after spatially targeted myocardial infarction: Neuropeptide and morphologic changes. Heart Rhythm, 12(5), pp.1027-1035.

Al-Gobari, M. et al., 2013. Beta-blockers for the prevention of sudden cardiac death in heart failure patients: a meta-analysis of randomized controlled trials. BMC cardiovascular disorders, 13, p.52.

Ando, M. et al., 2005. Efferent Vagal Nerve Stimulation Protects Heart Against IschemiaInduced Arrhythmias by Preserving Connexin43 Protein. Circulation, 112(2), pp.164170.

Anholt, T.A., Ayal, S. \& Goldberg, J.A., 2011. Recruitment and blocking properties of the CardioFit stimulation lead. Journal of neural engineering, 8(3), p.034004.

Ardell, J.L. et al., 1988. Differential sympathetic regulation of automatic, conductile, and contractile tissue in dog heart. The American journal of physiology, 255(5 Pt 2), pp.H1050-9.

Armour, J.A., 1996. Anatomy and funciton of peripheral autonomic neurons involved in cardiac regulation. In V. Shepard \& S. Vatner, eds. Nervous control of the heart. London, pp. 29-48.

Batulevicius, D., Pauziene, N. \& Pauza, D.H., 2005. Architecture and age-related analysis of the neuronal number of the guinea pig intrinsic cardiac nerve plexus. Annals of anatomy = Anatomischer Anzeiger : official organ of the Anatomische Gesellschaft, 187(3), pp.225-43.

Behling, A. et al., 2003. Cholinergic stimulation with pyridostigmine reduces ventricular arrhythmia and enhances heart rate variability in heart failure. American Heart Journal, 146(3), pp.494-500.

Binnie, C.D., 2000. Vagus nerve stimulation for epilepsy: a review. Seizure, 9(3), pp.161-9.

Bonnemeier, H. et al., 2003. Circadian profile of cardiac autonomic nervous modulation in healthy subjects: differing effects of aging and gender on heart rate variability. Journal of cardiovascular electrophysiology, 14(8), pp.791-9.

Bourke, T. et al., 2010. Neuraxial modulation for refractory ventricular arrhythmias: value of thoracic epidural anesthesia and surgical left cardiac sympathetic denervation.

Circulation, 121(21), pp.2255-62. 
Brack, K.E. et al., 2009. Direct evidence of nitric oxide release from neuronal nitric oxide synthase activation in the left ventricle as a result of cervical vagus nerve stimulation. The Journal of physiology, 587(Pt 12), pp.3045-54.

Brack, K.E. et al., 2007. Nitric oxide mediates the vagal protective effect on ventricular fibrillation via effects on action potential duration restitution in the rabbit heart. The Journal of physiology, 583(Pt 2), pp.695-704.

Brack, K.E., Coote, J.H. \& Ng, G.A., 2004. Interaction between direct sympathetic and vagus nerve stimulation on heart rate in the isolated rabbit heart. Experimental physiology, 89(1), pp.128-39.

Brack, K.E., Coote, J.H. \& Ng, G.A., 2010. Vagus nerve stimulation inhibits the increase in $\mathrm{Ca} 2+$ transient and left ventricular force caused by sympathetic nerve stimulation but has no direct effects alone--epicardial $\mathrm{Ca} 2+$ fluorescence studies using fura- $2 \mathrm{AM}$ in the isolated innervated beating rab. Experimental physiology, 95(1), pp.80-92.

Brack, K.E., Coote, J.H. \& Ng, G.A., 2011. Vagus nerve stimulation protects against ventricular fibrillation independent of muscarinic receptor activation. Cardiovascular Research, 91(3), pp.437-446.

Brack, K.E., Winter, J. \& Ng, G.A., 2013. Mechanisms underlying the autonomic modulation of ventricular fibrillation initiation--tentative prophylactic properties of vagus nerve stimulation on malignant arrhythmias in heart failure. Heart Failure Reviews, 18(4), pp.389-408.

Brodde, O.E. et al., 2001. Presence, distribution and physiological function of adrenergic and muscarinic receptor subtypes in the human heart. Basic research in cardiology, 96(6), pp.528-38.

Calvillo, L. et al., 2011. Vagal Stimulation, Through its Nicotinic Action, Limits Infarct Size and the Inflammatory Response to Myocardial Ischemia and Reperfusion. Journal of Cardiovascular Pharmacology, 58(5), pp.500-507.

Cao, J.M. et al., 2000. Relationship between regional cardiac hyperinnervation and ventricular arrhythmia. Circulation, 101(16), pp.1960-1969.

Casolo, G. et al., 1989. Decreased spontaneous heart rate variability in congestive heart failure. American Journal Of Cardiology, 64, pp.1162-1167.

Conlon, K. \& Kidd, C., 1999. Neuronal nitric oxide facilitates vagal chrontropic and dromotropic actions on the heart. Journal Of The Autonomic Nervous System, 75(2-3), pp.136-146.

Coote, J.H., 2013. Myths and realities of the cardiac vagus. The Journal of physiology, 591(Pt 17), pp.4073-4085.

Corr, P.B. \& Gillis, R.A., 1974. Role of the Vagus Nerves in the Cardiovascular Changes Induced by Coronary Occlusion. Circulation, 49(1), pp.86-97.

Dahlström, A. et al., 1965. Observations on adrenergic innervation of dog heart. The 
American journal of physiology, 209(4), pp.689-92.

Daubert, J.P. et al., 2006. Predictive value of ventricular arrhythmia inducibility for subsequent ventricular tachycardia or ventricular fibrillation in multicenter automatic defibrillator implantation trial (MADIT) II patients. Journal of the American College of Cardiology, 47(1), pp.98-107.

Deck, J. et al., 2005. Alpha7-nicotinic acetylcholine receptor subunit is not required for parasympathetic control of the heart in the mouse. Physiological genomics, 22(1), pp.86-92.

Dougherty, C.M. \& Burr, R.L., 1992. Comparison of heart rate variability in survivors and nonsurvivors of cardiac arrest. American Journal Of Cardiology, 70, pp.441-448.

Dvorakova, M. et al., 2005. Developmental changes in the expression of nicotinic acetylcholine receptor ?-subunits in the rat heart. Cell and Tissue Research, 319(2), pp.201-209.

Einbrodt, 1859. Ueber Herzeizung und ihr Verhaeltnis zum Blutdruck. Akademie der Wissenschaften (Vienna).Sitzungsberichte, 38, pp.345-359.

Eugenin, E.A. et al., 2003. TNF- Plus IFN- Induce Connexin43 Expression and Formation of Gap Junctions Between Human Monocytes/Macrophages That Enhance Physiological Responses. The Journal of Immunology, 170(3), pp.1320-1328.

De Ferrari, G.M. et al., 2011. Chronic vagus nerve stimulation: a new and promising therapeutic approach for chronic heart failure. European heart journal, 32(7), pp.84755 .

De Ferrari, G.M. et al., 1991. Vagal reflexes and survival during acute myocardial ischemia in conscious dogs with healed myocardial infarction. The American journal of physiology, 261(1 Pt 2), pp.H63-9.

De Ferrari, G.M. \& Schwartz, P.J., 2011. Vagus nerve stimulation: from pre-clinical to clinical application: challenges and future directions. Heart Failure Reviews, 16(2), pp.195-203.

Furchgott, R.F. \& Zawadzki, J. V, 1980. The obligatory role of endothelial cells in the relaxation of arterial smooth muscle by acetylcholine. Nature, 288(0028-0836 SB - M), pp.373-376.

Gao, P. et al., 2012. Prediction of Arrhythmic Events in Ischemic and Dilated Cardiomyopathy Patients Referred for Implantable Cardiac Defibrillator: Evaluation of Multiple Scar Quantification Measures for Late Gadolinium Enhancement Magnetic Resonance Imaging. Circulation: Cardiovascular Imaging, 5(4), pp.448-456.

Garfinkel, A. et al., 2000. Preventing ventricular fibrillation by flattening cardiac restitution. Proceedings Of The National Academy Of Sciences Of The United States Of America, 97(11), pp.6061-6066.

Gold, M.R. et al., 2016. Vagus Nerve Stimulation for the Treatment of Heart Failure: The INOVATE-HF Trial. Journal of the American College of Cardiology. 
Goldstein, R.E. et al., 1973. Influence of Atropine and of Vagally Mediated Bradycardia on the Occurrence of Ventricular Arrhythmias following Acute Coronary Occlusion in Closed-Chest Dogs. Circulation, 47(6), pp.1180-1190.

Grassi, G. et al., 2001. Sympathetic and reflex abnormalities in heart failure secondary to ischaemic or idiopathic dilated cardiomyopathy. Clinical science (London, England: 1979), 101(2), pp.141-6.

Grundy, D. \& Schemann, M., 2007. Enteric nervous system. Current Opinion in Gastroenterology, 23(2), pp.121-126.

Hamann, J.J. et al., 2013. Vagus nerve stimulation improves left ventricular function in a canine model of chronic heart failure. European journal of heart failure, 15(12), pp.1319-26.

Han, J. \& Moe, G.K., 1964. Nonuniform recovery of excitability in ventricular muscle. Circulation Research, 14(1), pp.44-60.

Han, S. et al., 2012. Electroanatomic remodeling of the left stellate ganglion after myocardial infarction. Journal of the American College of Cardiology, 59(10), pp.954961.

Haws, C.W. \& Burgess, M.J., 1978. Effects of bilateral and unilateral stellate stimulation on canine ventricular refractory periods at sites overlapping innervation. Circulation research, 42(2), pp.195-8.

Henning, R.J. et al., 2001. Vasoactive intestinal peptide: cardiovascular effects. Cardiovascular research, 49(1), pp.27-37.

Herring, N. et al., 2008. Neuropeptide $Y$ reduces acetylcholine release and vagal bradycardia via a $\mathrm{Y} 2$ receptor-mediated, protein kinase C-dependent pathway. Journal of molecular and cellular cardiology, 44(3), pp.477-85.

Herring, N., Golding, S. \& Paterson, D.J., 2000. Pre-synaptic NO-cGMP pathway modulates vagal control of heart rate in isolated adult guinea pig atria. Journal Of Molecular And Cellular Cardiology, 32(10), pp.1795-1804.

Hoover, D.B. et al., 2008. Novel neurotransmitters of the human intrinsic cardiac nervous system. The FASEB Journal, 22(1 Supplement), pp.1230.8-1230.8.

Ignarro, L.J. et al., 1987. Endothelium-derived relaxing factor produced and released from artery and vein is nitric oxide. Proc.Natl.Acad.Sci.U.S.A, 84, pp.9265-9269.

Ishise, H. et al., 1998. Time course of sympathovagal imbalance and left ventricular dysfunction in conscious dogs with heart failure. Journal of applied physiology (Bethesda, Md. : 1985), 84(4), pp.1234-41.

Johnson, T. a et al., 2004. Parasympathetic control of the heart. I. An interventriculo-septal ganglion is the major source of the vagal intracardiac innervation of the ventricles. Journal of applied physiology (Bethesda, Md. : 1985), 96(6), pp.2265-2272.

Jones, J.F.X., 2009. Cardiac defibrillator neurones. The Journal of physiology, 587(Pt 12), 
Jumrussirikul, P. et al., 1998. Interaction between neuronal nitric oxide synthase and inhibitory $G$ protein activity in heart rate regulation in conscious mice. The Journal of clinical investigation, 102(7), pp.1279-85.

Kalla, M. et al., 2016. Protection against ventricular fibrillation via cholinergic receptor stimulation and the generation of nitric oxide. The Journal of Physiology, 0, p.n/a-n/a.

Kammerling, J.J. et al., 1987. Denervation supersensitivity of refractoriness in noninfarcted areas apical to transmural myocardial infarction. Circulation, 76(2), pp.383-393.

Karma, A., 1993. Spiral breakup in model equations of action potential propagation in cardiac tissue. Physical Review Letters, 71(7), pp.1103-1106.

Katare, R.G. et al., 2010. Differential regulation of TNF receptors by vagal nerve stimulation protects heart against acute ischemic injury. Journal of Molecular and Cellular Cardiology, 49(2), pp.234-244.

Katare, R.G. et al., 2009. Vagal nerve stimulation prevents reperfusion injury through inhibition of opening of mitochondrial permeability transition pore independent of the bradycardiac effect. The Journal of Thoracic and Cardiovascular Surgery, 137(1), pp.223-231.

Kawano, H., Okada, R. \& Yano, K., 2003. Histological study on the distribution of autonomic nerves in the human heart. Heart and vessels, 18(1), pp.32-9.

Kelly, R.A., Balligand, J.L. \& Smith, T.W., 1996. Nitric-oxide and cardiac-function. Circulation Research, 79, pp.363-380.

Kent, K.M. et al., 1973. Electrical stability of acutely ischemic myocardium. Influences of heart rate and vagal stimulation. Circulation, 47(2), pp.291-298.

Kleiger, R.E. et al., 1987. Decreased heart rate variability and its association with increased mortality after acute myocardial infarction. American Journal Of Cardiology, 59, pp.256-262.

Klein, R.M. et al., 2000. [Inflammation of the myocardium as an arrhythmia trigger]. Zeitschrift für Kardiologie, 89 Suppl 3, pp.24-35.

Kolman, B.S., Verrier, R.L. \& Lown, B., 1975. The effect of vagus nerve stimulation upon vulnerability of the canine ventricle: role of sympathetic-parasympathetic interactions. Circulation, 52(4), pp.578-585.

Kolman, B.S., Verrier, R.L. \& Lown, B., 1976. The effect of vagus nerve stimulation upon vulnerability of the canine ventricle: Role of the sympathetic parasympathetic interactions. Circulation, 52, pp.578-585.

Koncz, I. et al., 2011. Electrophysiological effects of ivabradine in dog and human cardiac preparations: potential antiarrhythmic actions. European journal of pharmacology, 668(3), pp.419-26. 
Kumar, K. et al., 2000. Percutaneous nitroglycerin administration into the intact pericardial space suppresses ischemia-induced ventricular arrhythmias in closed-chest pigs. PACEPacing And Clinical Electrophysiology, 23(4(II)), p.730.

Kuo, C.S. et al., 1985. Mechanism of ventricular arrhythmias caused by increased dispersion of repolarization. European Heart Journal, 6, pp.63-70.

Kuo, K.S. et al., 1983. Characteristics and possible mechanism of ventricular arrhythmia dependent on the dispersion of action potential durations. Circulation, 67, pp.13561367.

Lee, D.S. et al., 2003. Effectiveness of implantable defibrillators for preventing arrhythmic events and death: A meta-analysis. Journal of the American College of Cardiology, 41(9), pp.1573-1582.

Levy, M.N., 1997. Neural control of cardiac function. Baillieres Clinical Neurology, 6, pp.227244.

Levy, M.N. et al., 1966. Sympathetic and parasympathetic interactions upon the left ventricle of the dog. Circulation Research, 19, pp.5-10.

Levy, M.N. \& Zieske, H., 1969. Autonomic control of cardiac pacemaker activity and atrioventricular transmission. Journal of Applied Physiology, 27(4), pp.465-470.

Li, M. et al., 2003. Vagal Nerve Stimulation Markedly Improves Long-Term Survival After Chronic Heart Failure in Rats. Circulation, 109(1), pp.120-124.

Li, W. \& Olshansky, B., 2011. Inflammatory cytokines and nitric oxide in heart failure and potential modulation by vagus nerve stimulation. Heart Failure Reviews, 16(2), pp.137145.

London, B. et al., 2003. Calcium-dependent arrhythmias in transgenic mice with heart failure. American journal of physiology. Heart and circulatory physiology, 284(2), pp.H431-41.

Mantravadi, R. et al., 2007. Autonomic nerve stimulation reverses ventricular repolarization sequence in rabbit hearts. Circulation research, 100(7), pp.e72-80.

Martins, J.B. \& Zipes, D.P., 1980. Effects of sympathetic and vagal nerves on recovery properties of the endocardium and epicardium of the canine left ventricle. Circulation Research, 46(1), pp.100-110.

Melgari, D. et al., 2015. hERG Potassium Channel Blockade by the HCN Channel Inhibitor Bradycardic Agent Ivabradine. Journal of the American Heart Association, 4(4), pp.e001813-e001813.

Morady, F. et al., 1988. Accentuated antagonism between beta-adrenergic and vagal effects on ventricular refractoriness in humans. Circulation, 77, pp.289-297.

Muscholl, E., 1980. Peripheral muscarinic control of norepinephrine release in the cardiovascular system. The American journal of physiology, 239(6), pp.H713-20. 
Myers, R.W. et al., 1974. Beneficial effects of vagal stimulation and bradycardia during experimental acute myocardial ischemia. Circulation, 49(5), pp.943-7.

Nattel, S. et al., 1981. Autonomic control of ventricular refractoriness. American Journal Of Physiology, 241, pp.H879-H882.

$\mathrm{Ng}$, G.A. et al., 2007. Autonomic modulation of electrical restitution, alternans and ventricular fibrillation initiation in the isolated heart. Cardiovascular Research, 73(4), pp.750-760.

$\mathrm{Ng}$, G.A. et al., 2009. Sympathetic nerve stimulation produces spatial heterogeneities of action potential restitution. Heart rhythm : the official journal of the Heart Rhythm Society, 6(5), pp.696-706.

Ng, G.A., 2014. Vagal modulation of cardiac ventricular arrhythmia. Experimental physiology, 99, pp.295-9.

Ng, G.A., Brack, K.E. \& Coote, J.H., 2001a. Differential effects of left and right vagus nerve stimulation on sinoatrial and atrioventricular nodes but not on ventricular electrophysiology - studies in the isolated rabbit heart with intact autonomic innervation. Journal of Physiology, 531P, p.182P-182P.

Ng, G.A., Brack, K.E. \& Coote, J.H., 2001b. Effects of direct sympathetic and vagus nerve stimulation on the physiology of the whole heart--a novel model of isolated Langendorff perfused rabbit heart with intact dual autonomic innervation. Experimental physiology, 86(3), pp.319-329.

Nolan, J. et al., 1998. Prospective study of heart rate variability and mortality in chronic heart failure - Results of the United Kingdom heart failure evaluation and assessment of risk trial (UK-Heart). Circulation, 98(15), pp.1510-1516.

Nolasco, J.B. \& Dahlen, R.W., 1968. A graphic method for the study of alternation in cardiac action potentials. J.Appl.Physiol, 25(2), pp.191-196.

Olshansky, B. et al., 2008. Parasympathetic Nervous System and Heart Failure: Pathophysiology and Potential Implications for Therapy. Circulation, 118(8), pp.863871.

Oppenheimer, S., 2006. Cerebrogenic cardiac arrhythmias: Cortical lateralization and clinical significance Stephen Oppenheimer. Clinical Autonomic Research, 16(1), pp.6-11.

Palmer, R.M., Ferrige, A.G. \& Moncada, S., 1987. Nitric oxide release accounts for the biological activity of endothelium-derived relaxing factor. Nature, 327, pp.524-526.

Panfilov, A. V. \& Holden, A. V., 1990. Self-generation of turbulent vortices in a twodimensional model of cardiac tissue. Physics Letters A, 151(1-2), pp.23-26.

Parker, G.W. et al., 1990. Central beta-adrenergic mechanisms may modulate ischemic ventricular fibrillation in pigs. Circulation Research, 66, pp.259-270.

Patel, V.H. et al., 2008. A novel method of measuring nitric-oxide-dependent fluorescence using 4,5-diaminofluorescein (DAF-2) in the isolated Langendorff-perfused rabbit heart. 
Pauza, D.H. et al., 2000. Morphology, distribution, and variability of the epicardiac neural ganglionated subplexuses in the human heart. Anat.Rec., 259, pp.353-382.

Pauza, D.H., Skripka, V. \& Pauziene, N., 2002. Morphology of the intrinsic cardiac nervous system in the dog: a whole-mount study employing histochemical staining with acetylcholinesterase. Cells, tissues, organs, 172(4), pp.297-320.

Pauziene, N. et al., 2016. Innervation of the rabbit cardiac ventricles. Journal of Anatomy, 228(1), pp.26-46.

Petkova-Kirova, P.S. et al., 2006. Electrical remodeling of cardiac myocytes from mice with heart failure due to the overexpression of tumor necrosis factor-alpha. American journal of physiology. Heart and circulatory physiology, 290(5), pp.H2098-107.

Pogwizd, S.M. \& Bers, D.M., 2004. Cellular basis of triggered arrhythmias in heart failure. Trends in cardiovascular medicine, 14(2), pp.61-6.

Potter, E.K. \& Ulman, L.G., 1994. Neuropeptides in sympathetic nerves affect vagal regulation of the heart. News In Physiological Sciences, 9, pp.174-177.

Premchand, R.K. et al., 2014. Autonomic regulation therapy via left or right cervical vagus nerve stimulation in patients with chronic heart failure: results of the ANTHEM-HF trial. Journal of cardiac failure, 20(11), pp.808-16.

Priori, S.G., Mantica, M. \& Schwartz, P.J., 1988. Delayed afterdepolarizations elicited in vivo by left stellate ganglion stimulation. Circulation, 78(1), pp.178-185.

Rabinowitz, S.H., Verrier, R.L. \& Lown, B., 1976. Muscarinic effects of vagosympathetic trunk stimulation on the repetitive extrasystole (RE) threshold. Circulation, 53(4), pp.622627.

Rajendran, P.S. et al., 2016. Myocardial infarction induces structural and functional remodelling of the intrinsic cardiac nervous system. The Journal of Physiology, 2, pp.321-341.

Randall, W.C. \& Rohse, W.G., 1956. The augmentor action of the sympathetic cardiac nerves. Circulation research, 4(4), pp.470-5.

Riccio, M.L., Koller, M.L. \& Gilmour, R.F., 1999. Electrical restitution and spatiotemporal organization during ventricular fibrillation. Circulation research, 84(8), pp.955-963.

La Rovere, M.T. et al., 1998. Baroreflex sensitivity and heart rate variability in prediction of total cardiac mortality after myocardial infarction. Lancet, 351, pp.478-484.

Rysevaite, K. et al., 2011. Immunohistochemical characterization of the intrinsic cardiac neural plexus in whole-mount mouse heart preparations. Heart rhythm : the official journal of the Heart Rhythm Society, 8(5), pp.731-8.

Sabbah, H.N. et al., 2011. Vagus nerve stimulation in experimental heart failure. Heart failure reviews, 16(2), pp.171-8. 
Saburkina, I. et al., 2010. Epicardial neural ganglionated plexus of ovine heart: Anatomic basis for experimental cardiac electrophysiology and nerve protective cardiac surgery. Heart Rhythm, 7(7), pp.942-950.

Samaan, A., 1935. The antagonistic cardiac nerves and heart rate. The Journal of physiology, 83(3), pp.332-40.

Scherlag, B.J. et al., 1970. Electrophysiology underlying ventricular arrhythmias due to coronary ligation. The American journal of physiology, 219(6), pp.1665-71.

Schwartz, P.J. et al., 1988. Autonomic mechanisms and sudden death - new insights from analysis of baroreceptor reflexes in conscious dogs with and without a myocardial infarction. Circulation, 78(4), pp.969-979.

Schwartz, P.J., 2014. Cardiac sympathetic denervation to prevent life-threatening arrhythmias. Nature reviews. Cardiology, 11(6), pp.346-53.

Schwartz, P.J. et al., 2004. Left Cardiac Sympathetic Denervation in the Management of High-Risk Patients Affected by the Long-QT Syndrome. Circulation, 109(15), pp.18261833.

Schwartz, P.J. et al., 1992. Prevention of Sudden Cardiac Death After a First Myocardial Infarction by Pharmacologic or Surgical Antiadrenergic Interventions. Journal of Cardiovascular Electrophysiology, 3(1), pp.2-16.

Schwartz, P.J., 1998. The autonomic nervous system and sudden death. European Heart Journal, 19, pp.F72-F80.

Schwartz, P.J., Billman, G.E. \& Stone, H.L., 1984. Autonomic mechanisms in ventricularfibrillation induced by myocardial ischemia during exercise in dogs with healed myocardial- infarction - an experimental preparation for sudden cardiac death. Circulation, 69(4), pp.790-800.

Schwartz, P.J., Snebold, N.G. \& Brown, A.M., 1976. Effects of unilateral cardiac sympathetic denervation on the ventricular fibrillation threshold. The American journal of cardiology, 37(7), pp.1034-40.

Shen, M.J. \& Zipes, D.P., 2014. Role of the autonomic nervous system in modulating cardiac arrhythmias. Circulation Research, 114(6), pp.1004-1021.

Sloan, R.P. et al., 2007. RR interval variability is inversely related to inflammatory markers: the CARDIA study. Molecular medicine (Cambridge, Mass.), 13(3-4), pp.178-84.

Taggart, P. et al., 2011. Anger, emotion, and arrhythmias: from brain to heart. Frontiers in physiology, 2, p.67.

Taggart, P. et al., 2003. Effect of adrenergic stimulation on action potential duration restitution in humans. Circulation, 107(2), pp.285-289.

Tracey, K.J., 2002. The inflammatory reflex. Nature, 420(6917), pp.853-859.

Ulphani, J.S. et al., 2010. Quantitative analysis of parasympathetic innervation of the porcine 
heart. Heart rhythm : the official journal of the Heart Rhythm Society, 7(8), pp.1113-9.

Vaillant, F. et al., 2008. Ivabradine induces an increase in ventricular fibrillation threshold during acute myocardial ischemia: an experimental study. Journal of cardiovascular pharmacology, 52(6), pp.548-54.

Vanoli, E. et al., 1991. Vagal stimulation and prevention of sudden death in conscious dogs with a healed myocardial infarction. Circulation Research, 68, pp.1471-1481.

Verrier, R.L., Thompson, P.L. \& Lown, B., 1974. Ventricular vulnerability during sympathetic stimulation: role of heart rate and blood pressure. Cardiovascular Research, 8, pp.602610.

Volders, P.G.A. et al., 2003. Probing the contribution of IKs to canine ventricular repolarization: key role for beta-adrenergic receptor stimulation. Circulation, 107, pp.2753-2760.

Wang, H. et al., 2003. Nicotinic acetylcholine receptor $\alpha 7$ subunit is an essential regulator of inflammation. Nature, 421(6921), pp.384-388.

Wang, S. et al., 2012. Activation of cardiac M3 muscarinic acetylcholine receptors has cardioprotective effects against ischaemia-induced arrhythmias. Clinical and experimental pharmacology \& physiology, 39(4), pp.343-9.

Wang, X. \& Gerdes, A.M., 1999. Chronic pressure overload cardiac hypertrophy and failure in guinea pigs: III. Intercalated disc remodeling. Journal of molecular and cellular cardiology, 31(2), pp.333-43.

Weiss, J.N. et al., 2000. Ventricular fibrillation: how Do We Stop the Waves From Breaking ? Circulation Research, 87(1524-4571 SB - IM), pp.1103-1107.

Wilde, A.A.M. et al., 2008. Left Cardiac Sympathetic Denervation for Catecholaminergic Polymorphic Ventricular Tachycardia. New England Journal Of Medicine, 358(19), pp.2024-2029.

Willich, S.N. et al., 1987. Circadian variation in the incidence of sudden cardiac death in the Framingham Heart Study population. The American journal of cardiology, 60(10), pp.801-6.

Winter, J. et al., 2012. Differential cardiac responses to unilateral sympathetic nerve stimulation in the isolated innervated rabbit heart. Autonomic neuroscience : basic \& clinical, 166(1-2), pp.4-14.

Yanowitz, F., Preston, J.B. \& Abildskov, J.A., 1966. Functional distribution of right and left stellate innervation to the ventricles. Production of neurogenic electrocardiographic changes by unilateral alteration of sympathetic tone. Circulation research, 18(4), pp.416-28.

Yoon, M.S. et al., 1977. Effects of vagal stimulation, atropine, and propranolol on fibrillation threshold of normal and ischemic ventricles. American heart journal, 93(1), pp.60-5.

Zannad, F. et al., 2015. Chronic vagal stimulation for the treatment of low ejection fraction 
heart failure: results of the NEural Cardiac TherApy foR Heart Failure (NECTAR-HF) randomized controlled trial. European heart journal, 36(7), pp.425-33.

Zanzinger, J., 1999. Role of nitric oxide in the neural control of cardiovascular function. Cardiovascular Research, 43, pp.639-649.

Zhang, C. et al., 2004. Effect of autonomic nervous system on the transmural dispersion of ventricular repolarization in intact canine. Journal of Huazhong University of Science and Technology. Medical sciences = Hua zhong ke ji da xue xue bao. Yi xue Ying De wen ban = Huazhong keji daxue xuebao. Yixue Yingdewen ban, 24(1), pp.37-40.

Zhang, Y. et al., 2009. Chronic vagus nerve stimulation improves autonomic control and attenuates systemic inflammation and heart failure progression in a canine high-rate pacing model. Circulation. Heart failure, 2(6), pp.692-9.

Zhang, Y.H., Zhu, J. \& Song, Y.C., 1998. Suppressing sympathetic activation with clonidine on ventricular arrhythmias in congestive heart failure. International journal of cardiology, 65(3), pp.233-8.

Zheng, C. et al., 2005. Vagal stimulation markedly suppresses arrhythmias in conscious rats with chronic heart failure after myocardial infarction. In 2005 IEEE Engineering in Medicine and Biology 27th Annual Conference. pp. 7072-7075.

Zhou, S. et al., 2004. Mechanisms of cardiac nerve sprouting after myocardial infarction in dogs. Circulation Research, 95(1), pp.76-83.

Zhou, S. et al., 2008. Spontaneous stellate ganglion nerve activity and ventricular arrhythmia in a canine model of sudden death. Heart rhythm : the official journal of the Heart Rhythm Society, 5(1), pp.131-9.

Zuanetti, G. et al., 1987. Protective effect of vagal stimulation on reperfusion arrhythmias in cats. Circulation Research, 61(3), pp.429-435. 


\section{Figure Legends}

Figure 1. Diagrammatic representation of APD restitution with A. action potential illustrating the timings of diastolic interval (DI) and APD at slow heart rate (long DI). B. With a faster heart rate with shorter DI, APD shortens and during restitution a small change in DI will lead to bigger changes in APD. A slowing of heart rate with the last DI being longer precedes a longer APD at the last beat. C. The relationship between APD and DI can be plotted which usually assumes a mono-exponential curve. The slope during APD restitution can be measured at the tangent or 1st derivative of the curve. The points corresponding to the situations in A. and B. are marked with the purple and blue symbols respectively.

Figure 2. A. Illustration of APD / DI relationship in the situation where the APD restitution slope is less than 1 . The top trace starts with 2 steady state beats, followed by a premature beat (with shorter DI) and subsequent rhythm at the same heart rate which means the sum of $A P D$ and $D I$ is constant and follows the straight line described by the equation $C L=A P D+$ DI. The premature beat with shorter DI gives rise to a shorter APD followed by a longer DI, as the APD restitution slope is shallow and less than 1 , the ensuing APD will be prolonged but to a limited extent, followed by a less shortened DI (compared to the previous beat) and so on where a steady state of stable APD and DI will be arrived. A. Illustration of APD / DI relationship in the situation where the APD restitution slope is greater than 1 . As opposed to A, the premature beat gives rise to a short DI and short APD, which following a same heart rate will give rise to a longer $\mathrm{DI}$, longer $\mathrm{APD}$ and then a much shorter DI (compared to the previous beat). A situation of alternating long and short APD will arise and the last stimulus lands on a long APD which blocked activation and can set of re-entry or wavebreak. 
Figure 3. Autonomic modulation of restitution, effective refractory period (ERP) and ventricular fibrillation threshold (VFT)

A. Standard restitution curves at baseline and during sympathetic (SS) and vagus nerve stimulation (VS) with maximum slopes indicated by dashed lines. B. First derivative of the fitted curves in A for maximum slope calculation. C. Effect of SS and VS on maximum slope of standard restitution, effective refractory period (ERP) and ventricular fibrillation threshold (VFT). D. Percentage change of data in C. Reproduced with permission from (Ng et al. 2007).

Figure 4. Regional variation of the effects of sympathetic nerve stimulation, expression of adrenergic nerves and ion channels Upper: Heterogeneity of restitution loops over the left ventricle. Restitution loops were measured during sympathetic nerve stimulation (SNS, open circles) and show shorter minimum action potential duration (APD) compared with pacing alone (pace, closed circles) at the Apex (A) and base (B). Lower left: Western blot comparing apex-base differences in KvLQT1 and tyrosine hydroxylase. Top: $\beta$-Actin controls. Middle: Tyrosine hydroxylase (TH a surrogate for sympathetic nerves is greater at the base. Bottom: KCNQ1 (KVLQT1), the pore forming main protein subunit responsible for IKS expressed higher at the base. Lower right: Density histograms comparing KCNQ1 and TH levels. Modified and reproduced with permission from (Ng et al. 2009).

Figure 5. The multifaceted anti-arrhythmic influence of vagus nerve stimulation. An illustration of the numerous anti-fibrillatory actions of the vagus nerve. (LEGEND: VNS= vagus nerve stimulation, $A P D=$ action potential duration, red=indirect effects, black=direct effects, $\leftrightarrow$ =interrelated factors) 
Figure 6. Nitric oxide mediates the anti-arrhythmic effect of vagus nerve stimulation (VNS)

$A \& B$. The effect of vagus nerve stimulation on electrical restitution $(A)$ and the restitution slope (B) and how this effect is abolished by perfusion with NG-nitro-L-arginine (L-NA) and restored by L-arginine (L-ARG). C-E) The effect of L-NA and L-NA \& L-Arg on VNS induced changes in restitution slope, effective refractory period (ERP) and ventricular fibrillation threshold (VFT) respectively. Reproduced with permission from (Brack et al. 2007).

Figure 7. Frequency dependent changes in NO fluorescence during vagal stimulation (VS)

A. Raw data illustrating continuous left ventricular pressure (LVP), aortic perfusion pressure (AP) and DAF-2 fluorescence at the excitation wavelength of $490 \mathrm{~nm}$ (F490) at varying intensities of VS. B. mean data showing the effect of varying intensities of VS on F490 and the change F490 (C). Reproduced with permission from (Brack et al. 2009).

Figure 8. Preservation of VNS-dependent changes in electrical restitution and NO fluorescence in the presence of atropine. Effect of atropine on the VNS-dependent changes in electrical restitution and maximal restitution slope. (A) Action potential duration restitution slopes at baseline, during vagus nerve stimulation (VNS) and VNS during perfusion with $0.1 \mathrm{mM}$ atropine. (B) Mean data representing the maximal restitution slope at baseline and during vagus nerve stimulation (VNS) during control, perfusion of $0.1 \mathrm{mM}$ atropine and washout. (C) Raw data illustrating left ventricular pressure (LVP), aortic perfusion pressure (AP), and DAF-2 fluorescence at the excitation wavelength of $490 \mathrm{~nm}$ (F490) with VNS during control and perfusion of $0.1 \mathrm{mM}$ atropine. (B) Mean F490 levels at baseline and during VNS during control, perfusion of $0.1 \mathrm{mM}$ atropine and washout. Modified and reproduced with permission from (Brack et al. 2011). 
Figure 1.

A

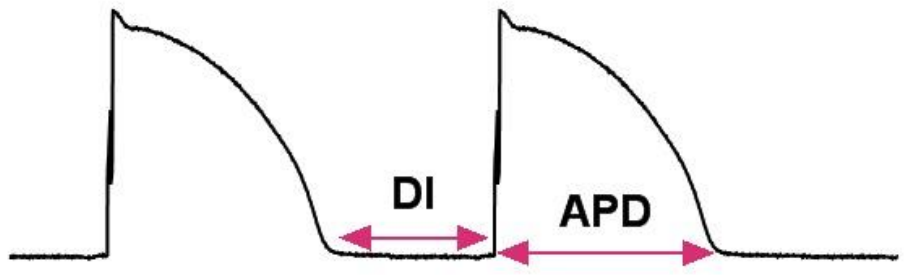

B

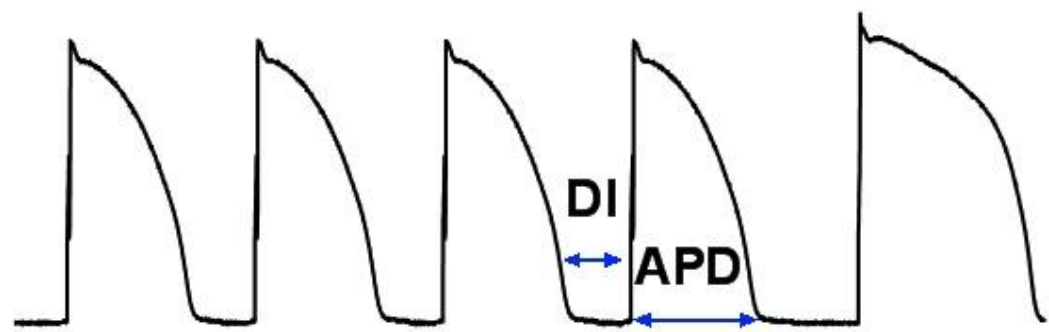

C

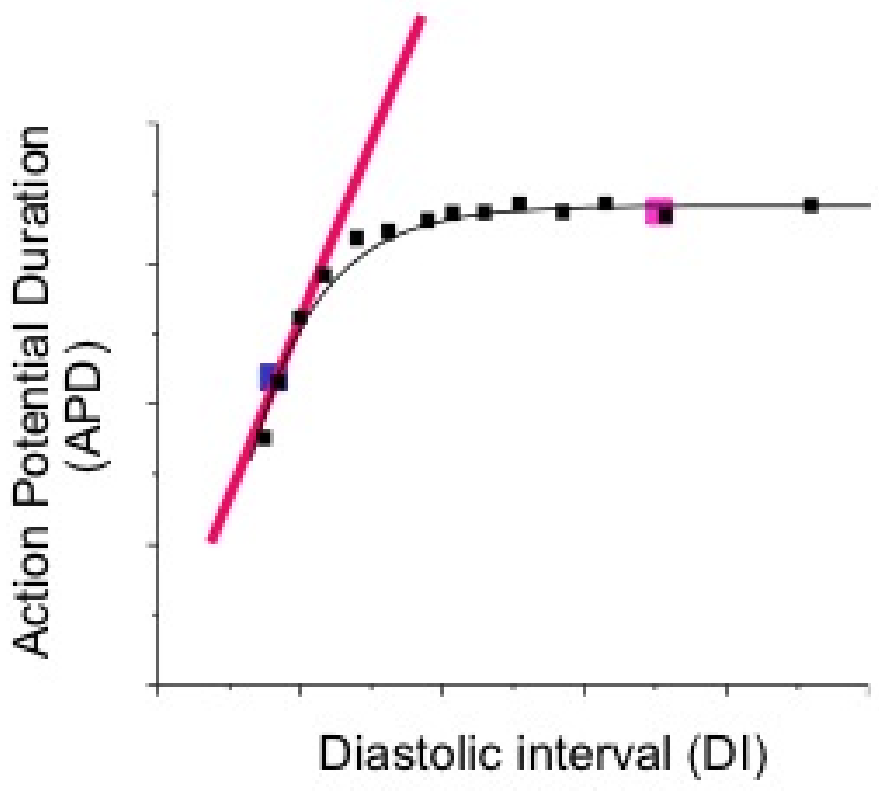

47

48

49

50

51

52

53

54

55

56

57

58

59

60

61

62 
Figure 2.

A
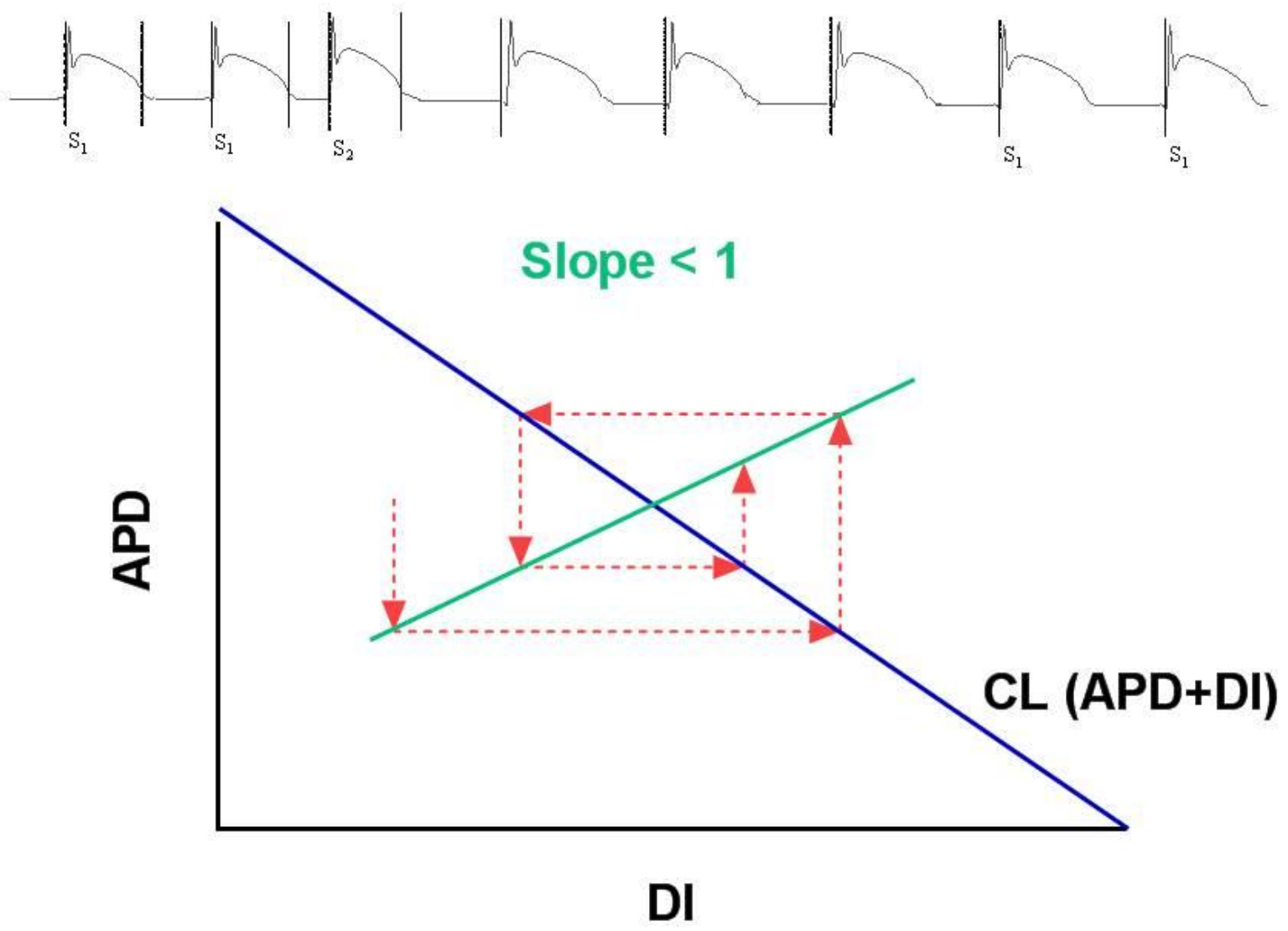

35

36

37

38

39

40

41

42

43

44

45

46

47

48

49

50

51

52

53

54

55

56

57

58

59

60

61

62 
B
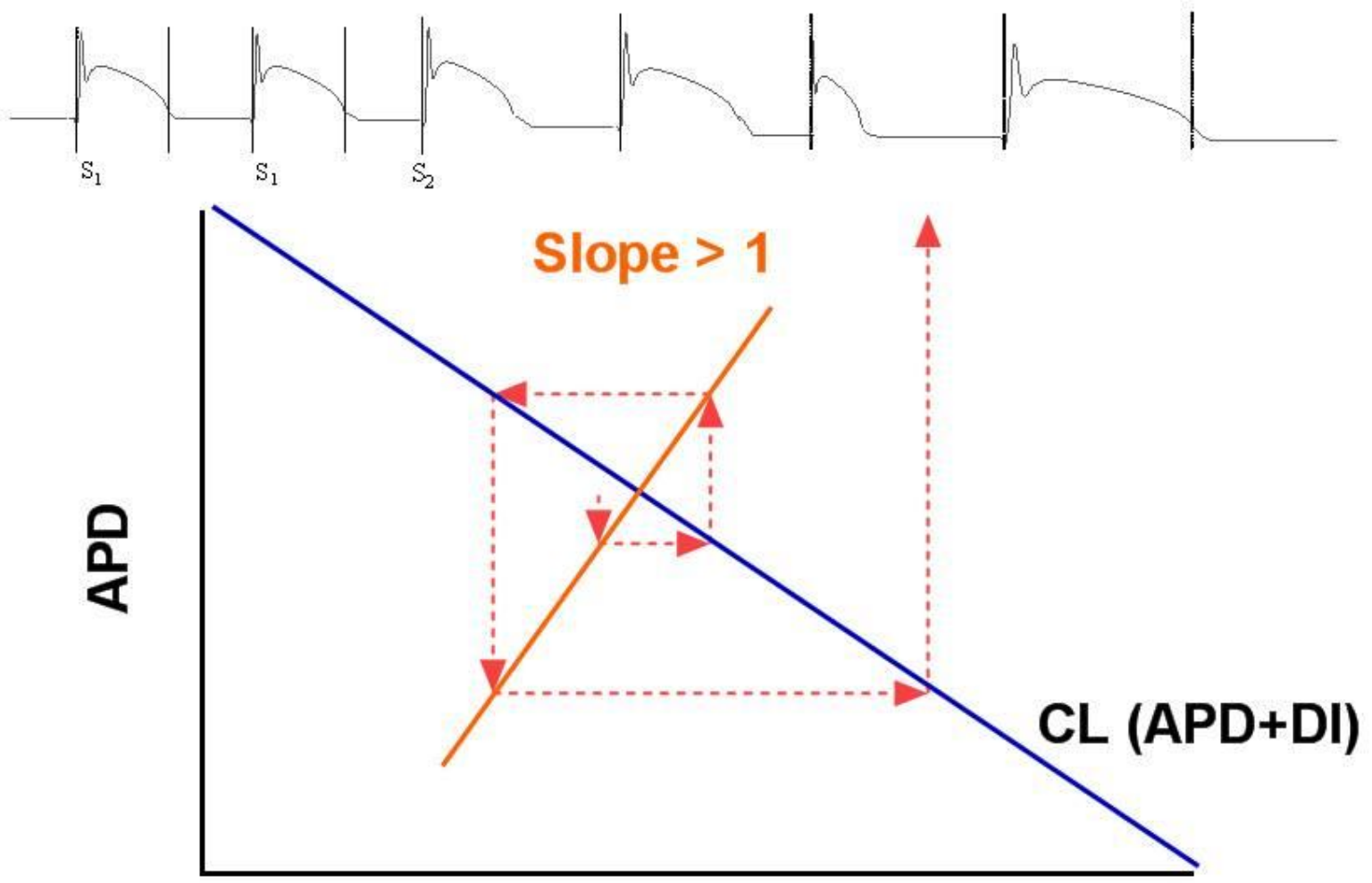

DI

35

36

37

38

39

40

41

42

43

44

45

46

47

48

49

50

51

52

53

54

55

56

57

58

59

60

61

62 
Figure 3.
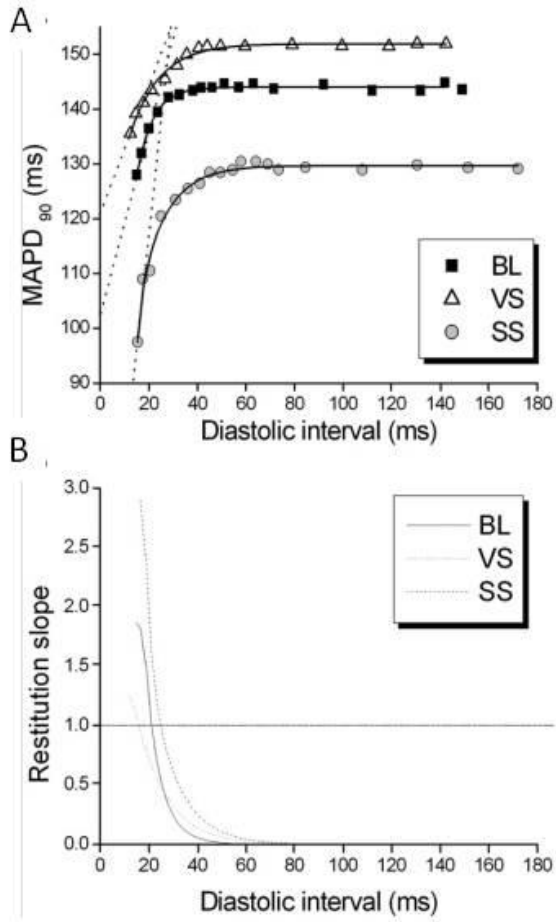

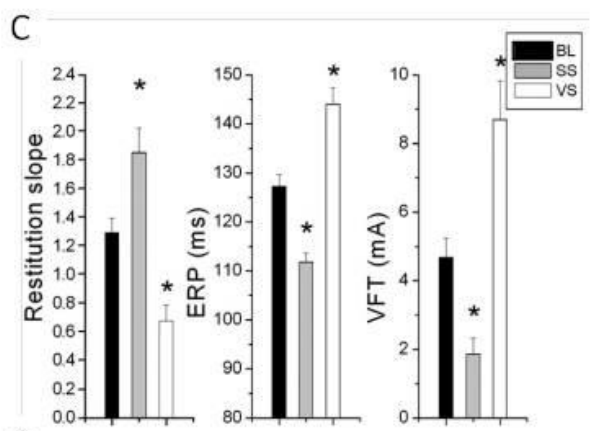

D

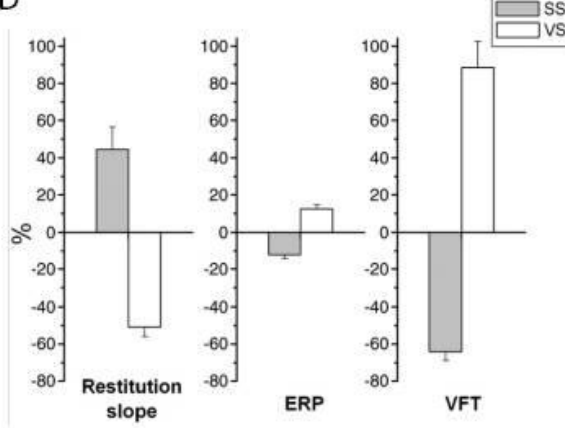


Figure 4.

A
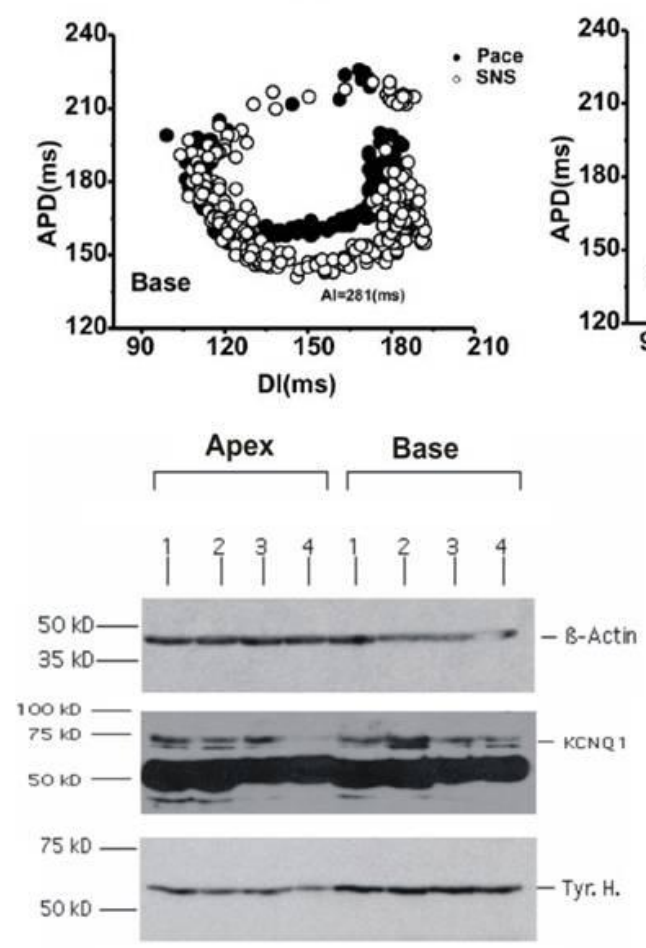

B

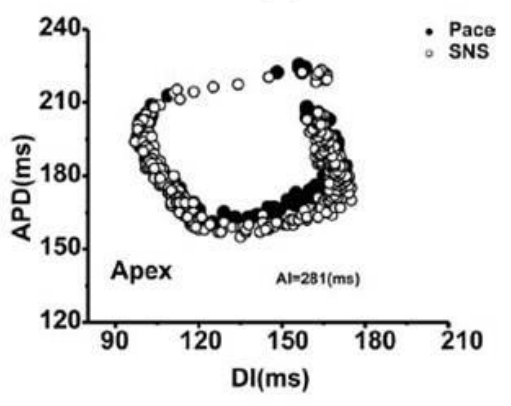

Apex Base

Mean values $n=4$

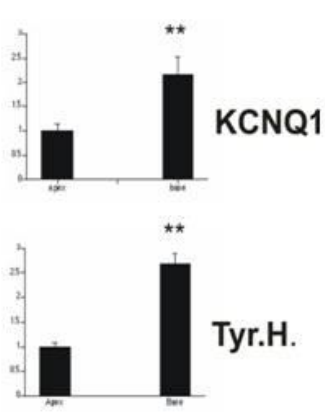


Figure 5.

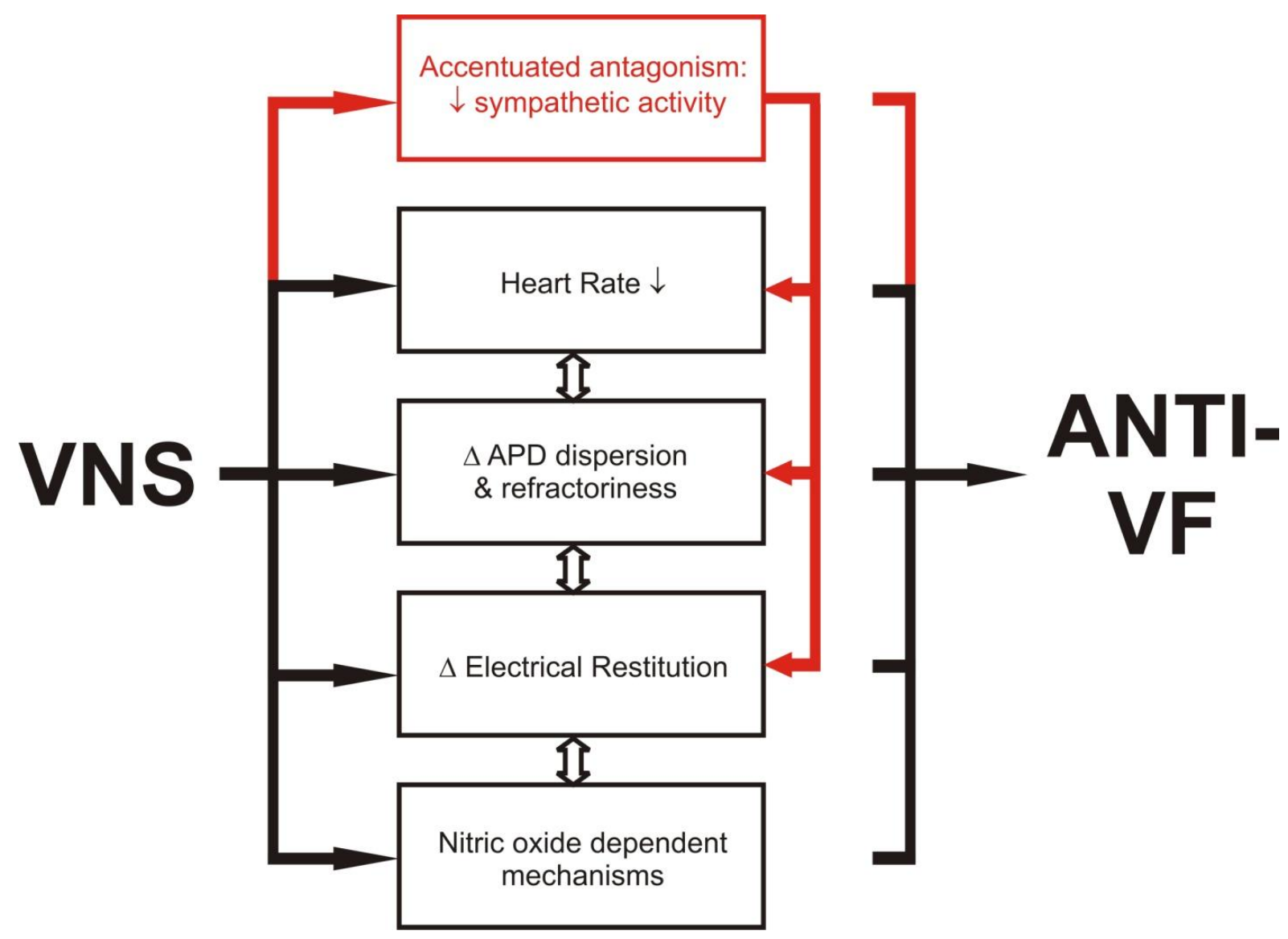


Figure 6.

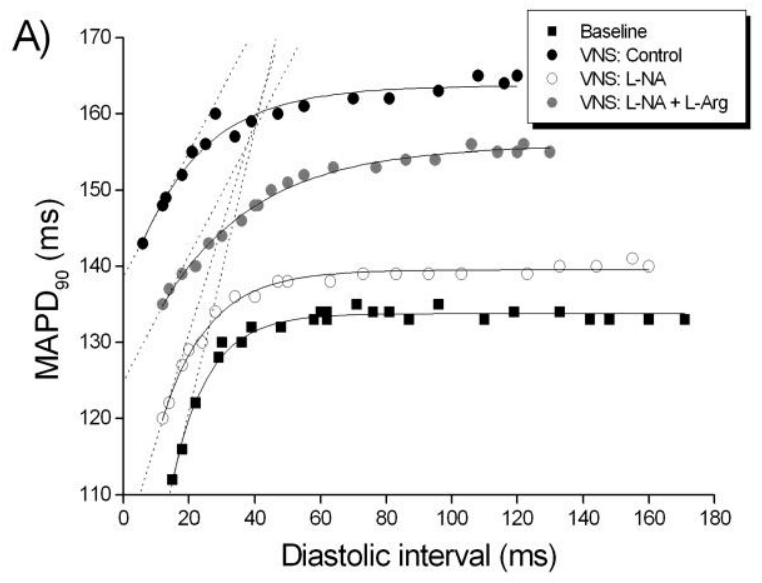

C)
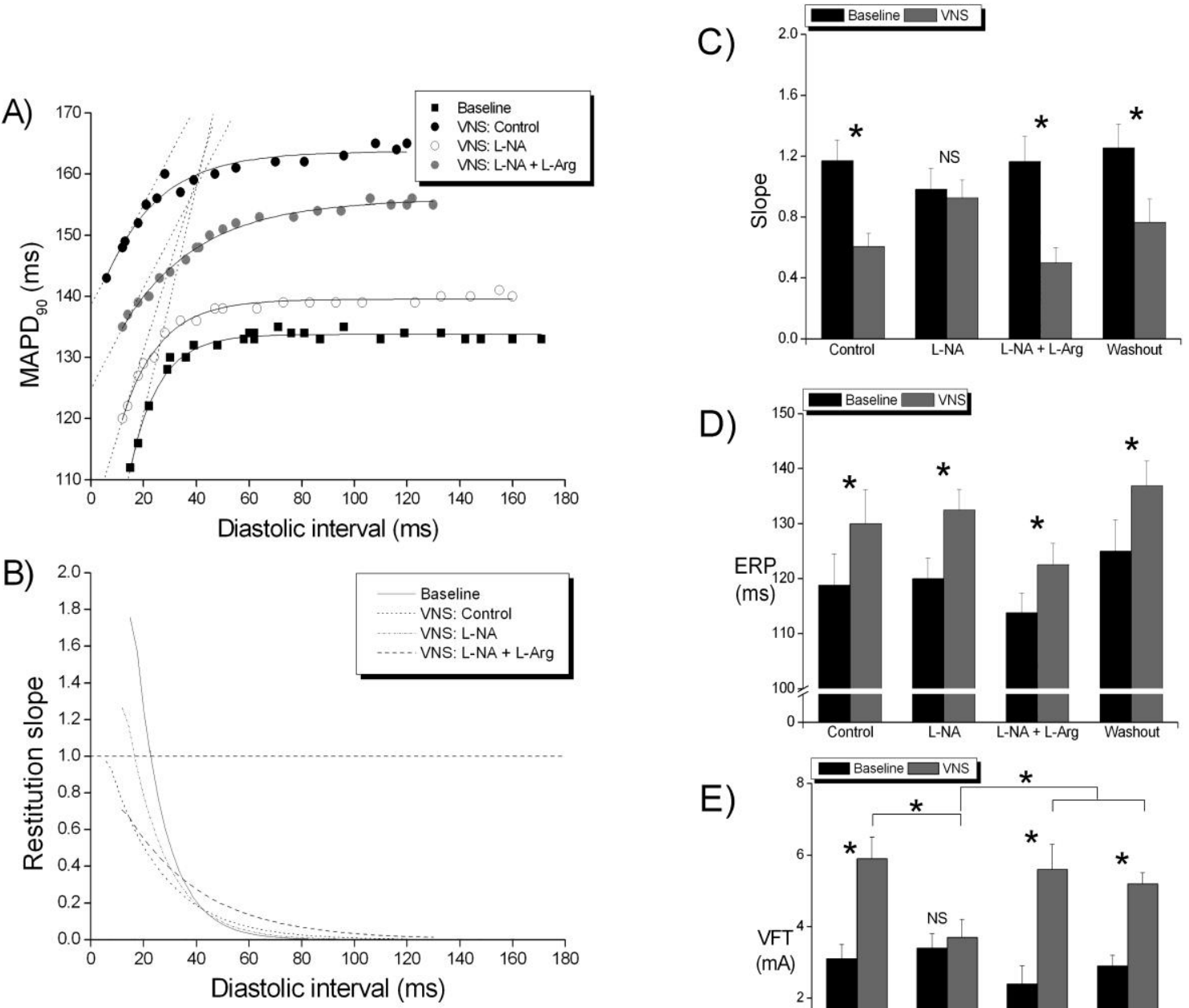

D)

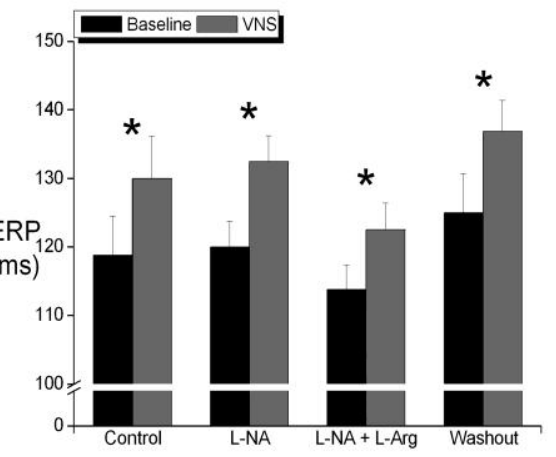

E)

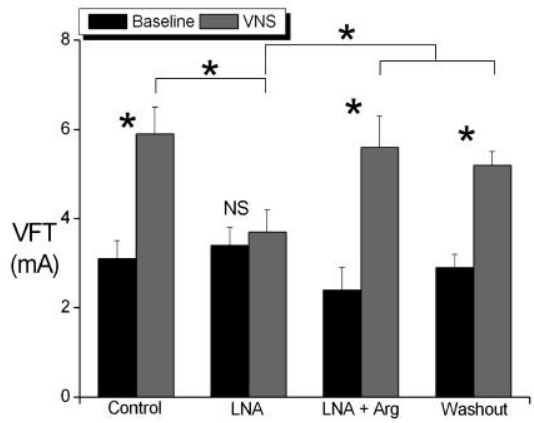


Figure 7.
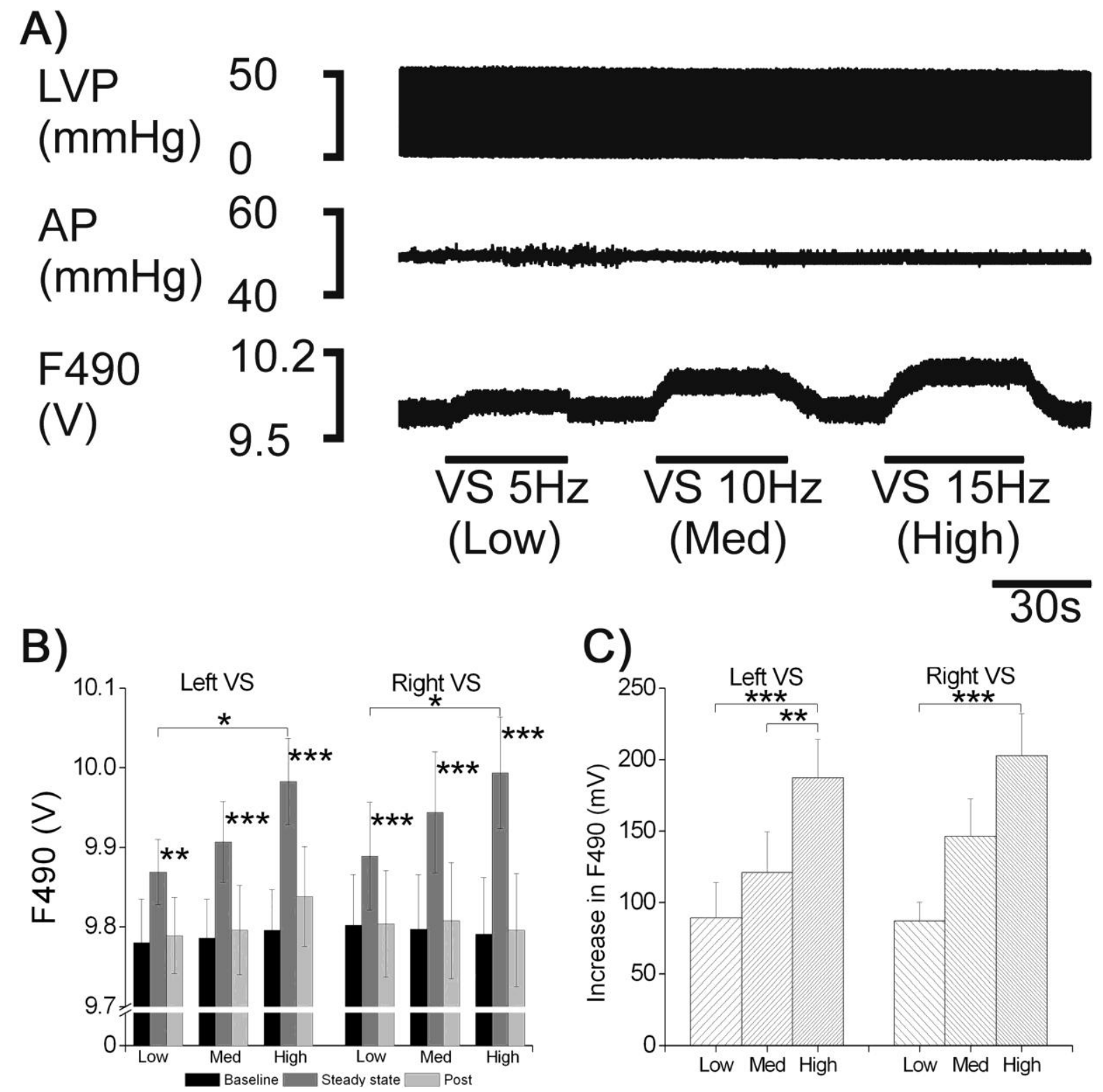
Figure 8.

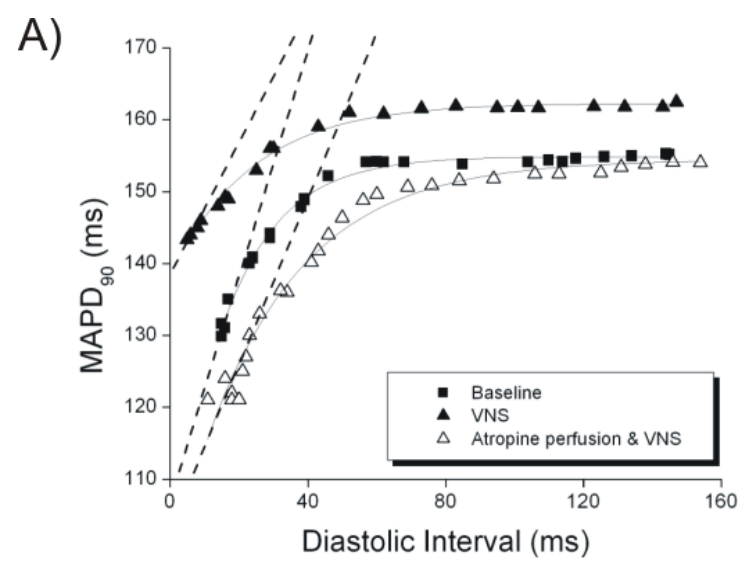

C)

B)

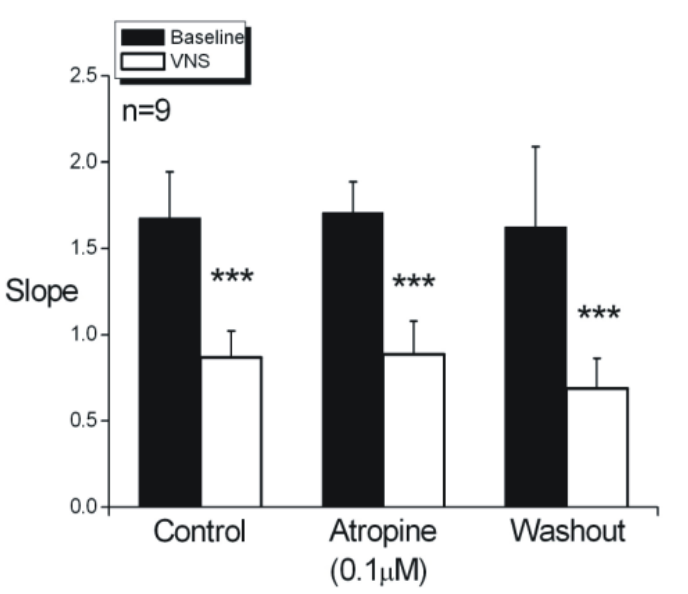

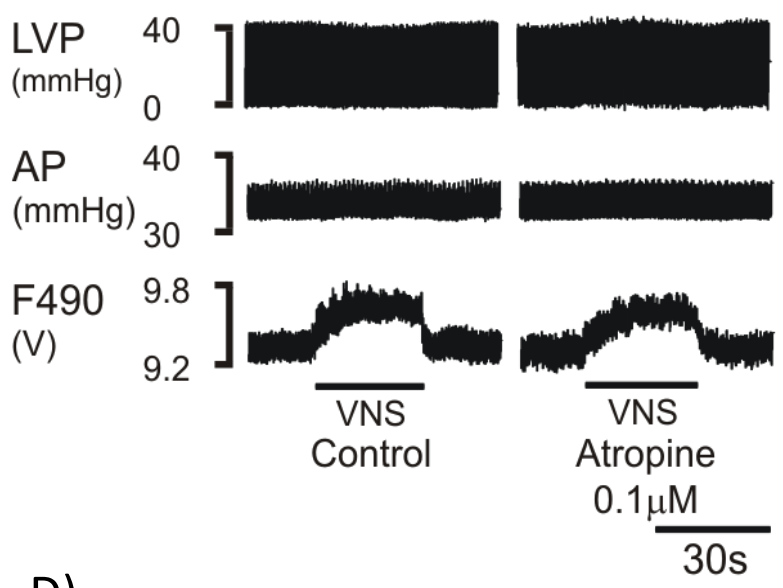

D)

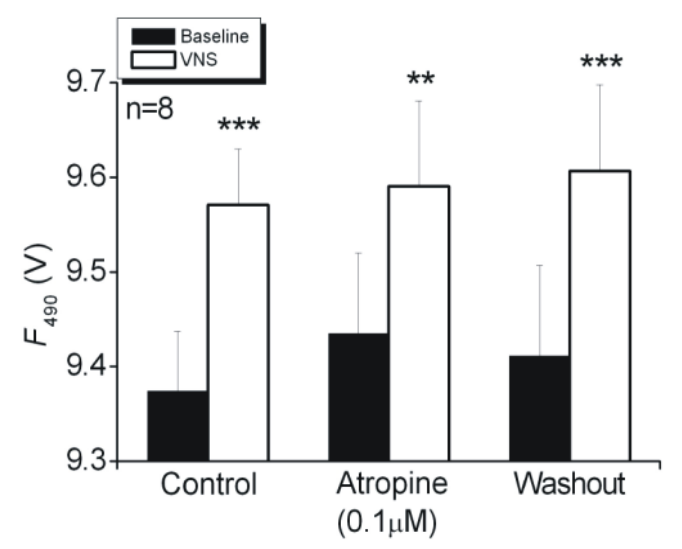

